Article

\title{
Modular Synthetic Approach to Carboranyl-Biomolecules Conjugates
}

\author{
Martin Kellert $\mathbb{Q}^{\circ}$, Jan-Simon Jeshua Friedrichs, Nadine Anke Ullrich, Alexander Feinhals $\mathbb{D}$, Jonas Tepper, \\ Peter Lönnecke and Evamarie Hey-Hawkins *(1)
}

Institute of Inorganic Chemistry, Faculty of Chemistry and Mineralogy, Leipzig University, Johannisallee 29, 04103 Leipzig, Germany; martin.kellert@uni-leipzig.de (M.K.); jf45guvu@studserv.uni-leipzig.de (J.-S.J.F.); nadine-ullrich6@web.de (N.A.U.); alexander.feinhals@gmx.de (A.F.); jonas.tepper@web.de (J.T.); loenneck@rz.uni-leipzig.de (P.L.)

* Correspondence: hey@uni-leipzig.de; Tel.: +49-341-97-36151

Citation: Kellert, M.; Friedrichs, J.-S.J.; Ullrich, N.A.; Feinhals, A.; Tepper, J.; Lönnecke, P.; Hey-Hawkins, E. Modular Synthetic Approach to Carboranyl-Biomolecules Conjugates. Molecules 2021, 26, 2057. https:// doi.org/10.3390/molecules26072057

Academic Editors: Andrea Trabocchi and Elena Lenci

Received: 15 March 2021

Accepted: 30 March 2021

Published: 3 April 2021

Publisher's Note: MDPI stays neutral with regard to jurisdictional claims in published maps and institutional affiliations.

Copyright: (c) 2021 by the authors. Licensee MDPI, Basel, Switzerland. This article is an open access article distributed under the terms and conditions of the Creative Commons Attribution (CC BY) license (https:/ / creativecommons.org/licenses/by/ $4.0 /)$.

\begin{abstract}
The development of novel, tumor-selective and boron-rich compounds as potential agents for use in boron neutron capture therapy (BNCT) represents a very important field in cancer treatment by radiation therapy. Here, we report the design and synthesis of two promising compounds that combine meta-carborane, a water-soluble monosaccharide and a linking unit, namely glycine or ethylenediamine, for facile coupling with various tumor-selective biomolecules bearing a free amino or carboxylic acid group. In this work, coupling experiments with two selected biomolecules, a coumarin derivative and folic acid, were included. The task of every component in this approach was carefully chosen: the carborane moiety supplies ten boron atoms, which is a tenfold increase in boron content compared to the L-boronophenylalanine (L-BPA) presently used in BNCT; the sugar moiety compensates for the hydrophobic character of the carborane; the linking unit, depending on the chosen biomolecule, acts as the connection between the tumor-selective component and the boron-rich moiety; and the respective tumor-selective biomolecule provides the necessary selectivity. This approach makes it possible to develop a modular and feasible strategy for the synthesis of readily obtainable boron-rich agents with optimized properties for potential applications in BNCT.
\end{abstract}

Keywords: boron neutron cancer therapy (BNCT); modular approach; carboxylic acids and amines

\section{Introduction}

Since boron neutron capture therapy (BNCT) was ascertained to be a very promising binary cancer treatment [1-4], research has focused on the development of potent and selective boron-containing drugs [5,6]. The main advantage of this therapy is the generation of highly cytotoxic particles comprising a high linear energy transfer (LET) ( $\alpha$ particle and Li particle). Their free mean path lengths of about 5 to $9 \mu \mathrm{m}[7,8]$ roughly represent the diameter of a human cell [5]; therefore, these particles can only harm the surrounding tissue within this radius. However, only the lighter isotope of boron, ${ }^{10} \mathrm{~B}$ (20\% natural abundance) [9], produces high LET particles after irradiation with thermal neutrons [10]. Therefore, BNCT agents have to be enriched with ${ }^{10} \mathrm{~B}[11,12]$. Activation of the BNCT agents is caused by irradiation with thermal neutrons $[13,14]$ for which ${ }^{10} \mathrm{~B}$ exhibits a large capture cross section (3835 barn, 1 barn $=1 \times 10^{-24} \mathrm{~cm}^{2}$ ) [9]. This renders BNCT a promising strategy to treat malignant tissue with tumor-selective boron-containing drugs [6,15-20], as the thermal neutron beam can be focused on the affected area [21-24], thus generating therapeutic particles only upon neutron irradiation. In this manner, normal tissue can be spared and severe side effects, as known from pure radiotherapy or systemic effective chemotherapy, can be reduced.

The first boron-containing compounds used in clinical trials were L-boronophenylalanine (L-BPA) and sodium borocaptate (BSH) $[5,6,10]$, but both compounds exhibit several drawbacks. For example, BSH and BPA do not exhibit optimized selectivity towards cancer 
cells (especially BSH), show a limited solubility in water (especially BPA [25]) and, in the case of $\mathrm{BSH}$, are not able to penetrate cells due to their anionic character. Therefore, their application follows a tailored strategy where BSH is mostly applied for glioma treatment, as the dianionic compound is able to cross the damaged blood-brain barrier adjoining the malignant tissue in the human brain, and BPA is used as its fructose complex to overcome the low water solubility $[5,6,10,25]$. Since May 2020, the company Stella Pharma [26] has been allowed to market Steboronine ${ }^{\circledR}$ [27] (generic name: Borofalan), which contains ${ }^{10} \mathrm{~B}$ enriched (99\%) L-BPA as its D-sorbitol complex. This BNCT agent, in comparison to the respective fructose complex, exhibits the advantage of being storable for about three years and does not have to be freshly prepared for each use with retention of its GMP grade.

Therefore, the development of novel boron-containing tumor-selective agents for application in BNCT is important to overcome these limitations $[19,20]$. For all compounds, the basic requirements that must be fulfilled are: sufficient water solubility, low inherent toxicity, high boron content and high tumor selectivity. Water solubility can be increased by using charged compounds [28] or introducing hydrophilic moieties [29,30]. Tumor-selectivity can be achieved by using essential biological nutrients, substrates like boronated saccharides or amino acids $[19,20,31]$, or even tumor-selective complex compounds, like boron-containing antibodies [20,29,30,32-36]. A variety of different boroncontaining bioconjugates are known, including nucleosides [16], carbohydrates [37,38], amino acids [39-41] and peptides [29,30,42,43]. One main prerequisite of BNCT especially plays an essential role in this treatment, namely the selective accumulation of sufficient amounts of ${ }^{10} \mathrm{~B}$-containing compounds in cancerous tissues, so that the therapeutically active particles destroy only the malignant cells without destroying healthy tissue. An effective treatment requires boron concentrations of $10-30 \mu \mathrm{g}{ }^{10} \mathrm{~B} / \mathrm{g}$ tumor, or $10^{9}{ }^{10} \mathrm{~B}$ atoms/cancer cell $[7,10]$. One approach focuses on compounds with a very large boron content $[29,30,42,44,45]$, another on the use of very selective BNCT agents over a longer period, taking advantage of specific shuttle systems that facilitate accumulation of the compound in the cells by internalization processes $[17,29,30,33,46]$. We pursued a combination of both strategies by combining tumor-selective small peptides, such as highly selective $\mathrm{G}$ protein-coupled receptor agonists, as biomolecules with meta-carborane derivatives to increase the boron load [29,30,43,47,48]. However, very high carborane loading (more than two carboranes attached to a peptide including 36 amino acids) results in loss of solubility or aggregation in aqueous media and, therefore, decreased potency and higher $\mathrm{EC}_{50}$ values $[29,30]$. Carbohydrate moieties, such as galactosyl groups, can be employed to compensate the hydrophobic character of carborane clusters (up to eight modified carboranes attached to the same peptide comprising 36 amino acids).

Here, we report the development of small molecules representing potential boronrich coupling partners for tumor-selective molecules based on a modular strategy [47-49] combining readily available starting materials, like meta-carborane, $\alpha$-D-galactopyranose and glycine or ethylenediamine derivatives (compounds 5 and $\mathbf{6}$ in Scheme 1). Compounds bearing a primary amine or carboxylic acid group represent potentially universal coupling partners for biomolecules [48]; representative coupling experiments are also included here to demonstrate the generalizability of this approach. 


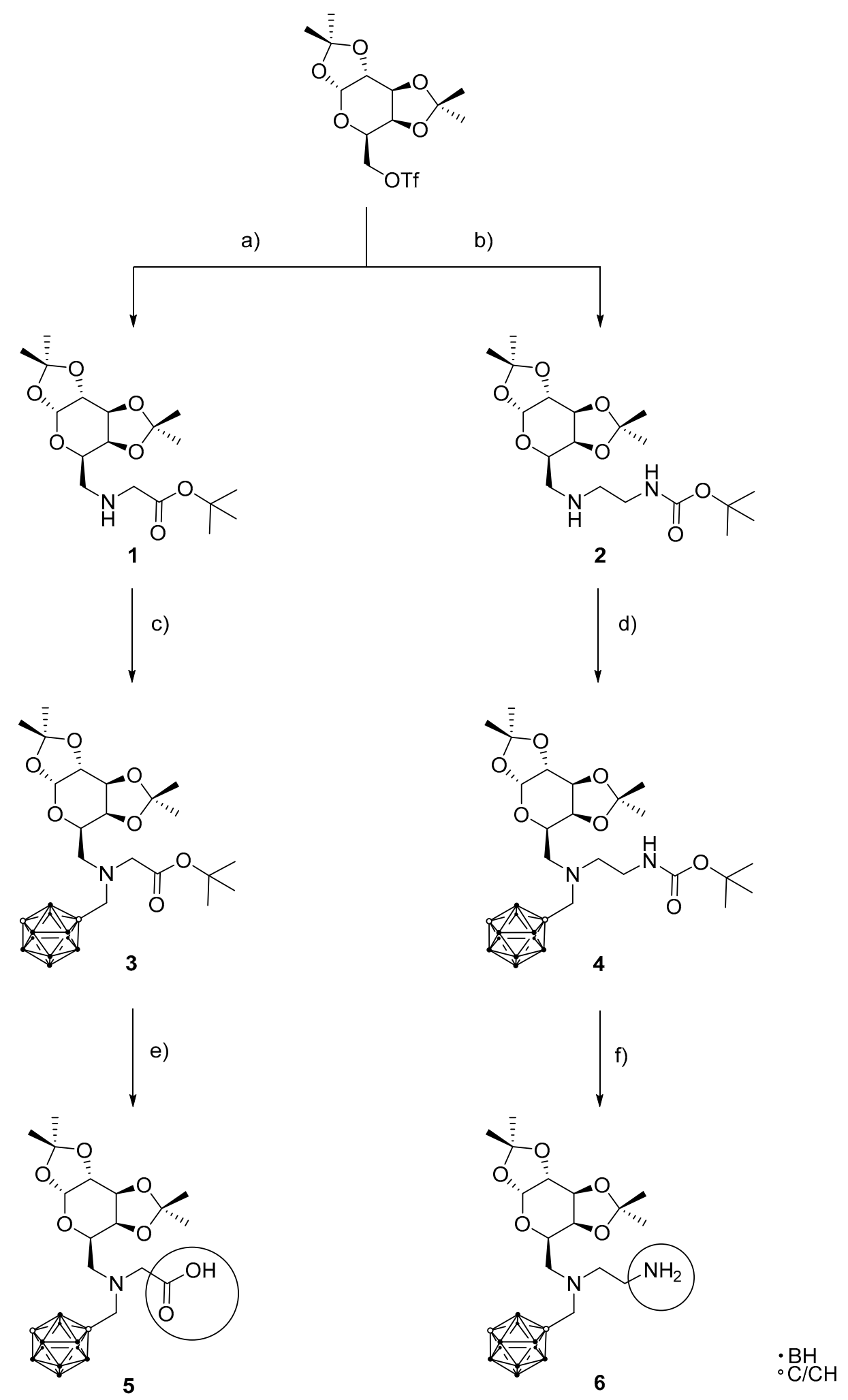

Scheme 1. Synthesis of galactopyranosyl-modified meta-carborane-containing carboxylic acid (5) and primary amine (6) as the target compounds for modular conjugation with tumor-selective biomolecules. (a) 1.20 eq. tert-butyl glycinate hydrochloride, 2.96 eq. DIPEA, MeCN, 72 h, $45{ }^{\circ} \mathrm{C}, 68 \%$; (b) 1.11 eq. N-tert-butoxycarbonyl-ethylenediamine, 1.12 eq. DIPEA, MeCN, $15 \mathrm{~min}, 0^{\circ} \mathrm{C}$, then $48 \mathrm{~h}, 40{ }^{\circ} \mathrm{C}, 77 \%$; (c) 1.20 eq. 1-(trifluoromethanesulfonylmethyl)-1,7-dicarba-closododeca-borane(12), 1.20 eq. $\mathrm{K}_{2} \mathrm{CO}_{3}$, toluene, $43 \mathrm{~h}, 95{ }^{\circ} \mathrm{C}, 54 \%$; (d) 1.20 eq. 1-(trifluoromethanesulfonylmethyl)-1,7-dicarbacloso-dodecaborane(12), 1.20 eq. $\mathrm{K}_{2} \mathrm{CO}_{3}$, toluene, $48 \mathrm{~h}, 98{ }^{\circ} \mathrm{C}, 51 \%$; (e) 55.0 eq. TFA, $3 \mathrm{~h}, \mathrm{rt}, 65 \%$; (f) 48.2 eq. TFA, $3 \mathrm{~h}$, rt, quant. 
In this regard, the synthesis of bifunctional anticancer drugs is of special interest. Several examples are known where drugs are used as theranostic compounds [50,51] or exhibit dual effects $[52,53]$. For example, derivatives of 7-amino-4-methylcoumarin are known for their anticancer activity [54]. Thus, combination with a carborane derivative can lead to drugs that possess anti-cancer properties and the ability to capture thermal neutrons for applications in BNCT. Furthermore, folic acid and its derivatives, which are already used as tumor-selective synthons for applications in BNCT [15,32,55], can act as diagnostic probes for some solid cancer types when combined with imaging agents [56]. Thus, conjugates of folic acid with carborane derivatives and an imaging agent using both carboxylic groups of folic acid could be used to generate highly selective BNCT agents with excellent imaging properties.

\section{Results and Discussion}

The starting materials 1-(trifluoromethanesulfonylmethyl)-1,7-dicarba-closo-dodeca borane(12) and 1,2:3,4-di-O-isopropylidene-6-deoxy- $\alpha$-D-galactopyranosyl-6-triflate were prepared according to protocols from the literature. The synthetic procedures of Goto and co-workers for the synthesis of 1-(hydroxymethyl)-1,7-dicarba-closo-dodecaborane(12) [57] and those of Kalinin and co-workers for the preparation of 1-(trifluoromethanesulfonylmeth yl)-1,7-dicarba-closo-dodecaborane(12) [58] were employed. 1,2:3,4-Di-O-isopropylidene- $\alpha$ D-galactopyranose is commercially available or can be prepared in quantitative yield according to a procedure described by Saltan and co-workers [59]. 1,2:3,4-Di-O-isopropylidene$\alpha$-D-galactopyranose was converted to the triflate 1,2:3,4-di-O-isopropylidene-6-deoxy$\alpha$-D-galactopyranosyl-6-triflate following a procedure described by Brackhagen and coworkers [60].

The next step was the introduction of the respective linking units (glycine or ethylenediamine) starting from the commercially available protected derivatives, namely tert-butyl glycinate hydrochloride and $\mathrm{N}$-tert-butoxycarbonyl-ethylenediamine, to prevent undesired side reactions during the synthetic steps. The reaction with the galactopyranosyl moiety was conducted under basic conditions using $\mathrm{N}, \mathrm{N}$-diisopropylethylamine (DIPEA) as a base in acetonitrile $(\mathrm{MeCN})$ at elevated temperature (Scheme 1a,b). Both reactions gave the desired products, tert-butyl- $N$-(1,2:3,4-di-O-isopropyliden-6-deoxy- $\alpha$-D-galactopyranos-6yl)glycinate (1) and tert-butyl-\{2-[(1,2:3,4-di-O-isopropylidene-6-deoxy- $\alpha$-D-galactopyranos6-yl)amino]ethyl\}carbamate (2) in good to excellent yields.

Beside the desired product 2, the disubstituted compound tert-butyl-(2-\{[bis $(1,2: 3,4$ di-O-isopropylidene-6-deoxy- $\alpha$-D-galactopyranos-6-yl)]amino\}ethyl)-carbamate ( $\left.2^{\prime}\right)$ was isolated with an $8 \%$ yield (see the Supplementary Materials). This product was formed due to the increased nucleophilicity of the secondary amine in $\mathbf{2}$ in comparison to a primary amine in the starting material; the amount corresponded to the small excess of the ethylenediamine derivative employed here.

The carboranyl moiety was introduced by reacting 1 and 2 with 1-(trifluoromethanesulf onylmethyl)-1,7-dicarba-closo-dodecaborane(12) under basic conditions (potassium carbonate) in toluene (Scheme 1c,d); for optimized reaction conditions see the Supplementary Materials. The products, tert-butyl- $N$-[(1,7-dicarba-closo-dodecaboran-1-yl)methyl]- $N$-(1,2:3,4di-O-isopropylidene-6-deoxy- $\alpha$-D-galacatopyranos-6-yl)glycinate (3) and tert-butyl-\{2-[(1,7dicarba-closo-dodecaboran-1-ylmethyl)-(1,2:3,4-di-O-isopropyliden-6-deoxy- $\alpha$-D-galactopy ranos-6-yl)amino]ethyl\}carbamat (4) were obtained in moderate yields (54 and $51 \%$, respectively), presumably due to steric hindrance (bulky carboranyl moiety and secondary amine derivative).

The final step was the deprotection of the acid-labile protecting groups $\left(\mathrm{R}(\mathrm{CO}) \mathrm{O}^{t} \mathrm{Bu}\right.$ and $\mathrm{RNH}(\mathrm{CO}) \mathrm{O}^{t} \mathrm{Bu}$, where $\mathrm{R}$ is the organic moiety) of compounds 3 and 4 with trifluoroacetic acid (TFA), with formation of the volatile reaction products isobutene and carbon dioxide leading to very pure products $N$-[(1,7-dicarba-closo-dodecaboran-1-yl)methyl]$N$-(1,2:3,4-di-O-isopropylidene-6-deoxy- $\alpha$-D-galacatopyranos-6-yl)glycine (5) and $N^{1}$-(1,7dicarba-closo-dodecaboran-1-yl-methyl)- $N^{1}$-(1,2:3,4-di-O-isopropylidene-6-deoxy- $\alpha$-D-galac 
topyranos-6-yl)ethane-1,2-diamine (6) in high yields due to a facile purification [61]. Anhydrous conditions must be employed to prevent cleavage of the monosaccharide protecting groups [61]. Compound 5 was first purified by column chromatography, which led to a noticeable loss of product ( $47 \%$ yield) due to the highly polar carboxylic acid group. Therefore, compounds $\mathbf{5}$ and $\mathbf{6}$ were purified by evaporation of all volatile reaction products with dichloromethane (DCM) as an entrainer, leading to pure compounds in good to excellent yields (65\% and quantitative yield, respectively; details are given in the Supplementary Materials). Apparently, deprotection of the carboxylic acid ester (tert-butyl group) is less efficient than deprotection of the carbamate (Boc group).

The new compounds 1-6 were fully characterized by NMR spectroscopy (numbering scheme given in Figure S1), mass spectrometry and infrared spectroscopy and showed a purity of at least $95 \%$. Furthermore, we were able to demonstrate that the same procedure can also be applied for the synthesis of the corresponding ortho-carborane derivative $N^{1}$-[(1,2-dicarba-closo-dodecaboran-1-yl)methyl]- $N^{1}$-(1,2:3,4-di-O-isopropylidene-6deoxy- $\alpha$-D-galactopyranos-6-yl)ethane-1,2-diamine (see the Supplementary Materials for further details).

With the glycine derivative 5 and ethylenediamine derivative 6 in hand, exemplary coupling reactions with two selected biomolecules that play roles in cancer treatment were conducted. 7-Amino-4-methylcoumarin (Figure 1, left), already employed in the preparation of polyfunctional cancer therapeutics based on cisplatin derivatives [62] but not for the preparation of potential BNCT agents [63-65], was selected as a coupling partner for 5. As coupling partner for amine 6, folic acid (Figure 1, right) was chosen as this biomolecule has already been employed in the synthesis of potential BNCT agents $[15,32,46,55,66]$. Some cancer types overexpress folate receptors on their cell membrane surfaces, which can be used for selective uptake of the final BNCT agent in the respective cancer cells, increasing the efficacy of the therapy $[32,67]$.<smiles>Cc1cc(=O)oc2cc(N)ccc12</smiles><smiles>Nc1nc(O)c2nc(CNc3ccc(C(=O)NC(CCC(=O)O)C(=O)O)cc3)cnc2n1</smiles>

Figure 1. 7-Amino-4-methylcoumarin (left) and folic acid (right) as two biomolecules selected to demonstrate the coupling behavior of glycine derivative 5 and ethylenediamine derivative 6 .

The strategy for coupling glycine derivative 5 with the weakly nucleophilic coumarin derivative followed a procedure described by Quéléver and co-workers using phosphoryl chloride and pyridine [68] and resulted in $N^{1}$-[(1,7-dicarba-closo-dodecaborane-1yl)methyl]- $N^{1}$-(1,2:3,4-di-O-isopropylidene-6-deoxy- $\alpha$-D-galacatopyranos-6-yl)- $N^{2}$-[(4-meth yl)-2-oxo-2H-chromen-7-yl)glycineamide (7), albeit in low yield (27\%) (Scheme 2). Attempts to use less harsh conditions ( $N, N^{\prime}$-dicyclohexylcarbodiimide (DCC) and $N$-hydroxysuccini mide (NHS)) for this coupling reaction were not successful [69]. Using different coupling reagents (1-ethyl-3-(3-dimethylaminopropyl)carbodiimide (EDCI) and hydroxybenzotriazol (HOBt) with $N, N$-diisopropylethylamine (DIPEA) as base [69]) yielded the desired product; however, it was in a very low yield of only $18 \%$, indicating that this method is inferior to the phosphoryl chloride approach and the low reactivity of the coumarin derivative is the main issue. Compound 7 was fully characterized by NMR spectroscopy, mass spectrometry and infrared spectroscopy, proving the successful synthesis (with at least $95 \%$ purity) of this bioconjugate as a proof of principle in this approach. 

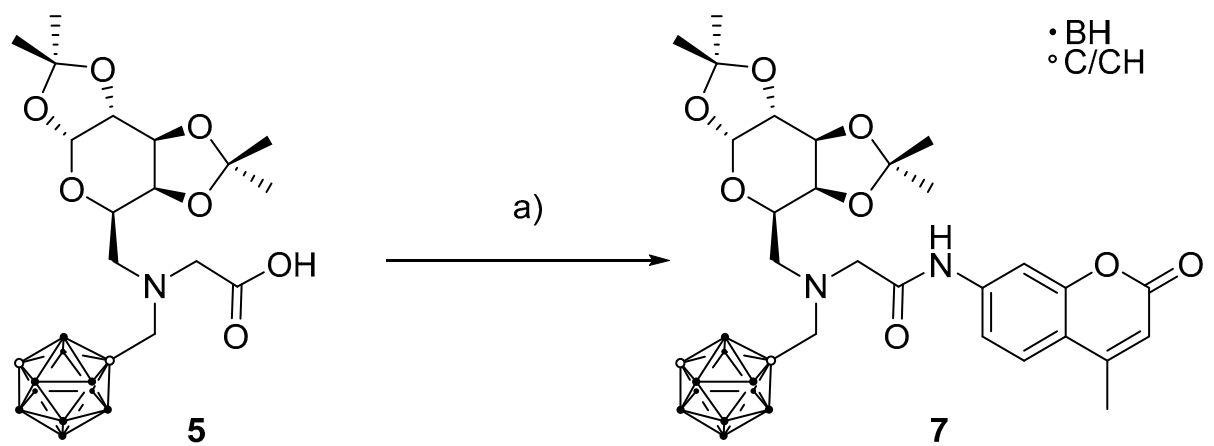

Scheme 2. (a) 1.20 eq. 7 -amino-4-methylcoumarin, 1.10 eq. $\mathrm{POCl}_{3}$, pyridine, $1 \mathrm{~h},-18{ }^{\circ} \mathrm{C}, 27 \%$.

The coupling reaction between folic acid and the primary amine 6 turned out to be more complicated due to the presence of two unprotected carboxylic acid groups in the former. Similar reactions have been reported where no additional protecting group was used for the secondary carboxylic acid group [55,66,70], as the primary $\mathrm{COOH}$ group exhibits a higher reactivity and, therefore, is more prone to undergo coupling reactions. However, here, the reaction of $\mathbf{6}$ with folic acid, following the procedure described by Trindade and coworkers, using DCC and NHS as activation reagents [70], gave both the desired monosubstituted species, namely $N^{2}-(4-\{[(2-a m i n o-4-h y d r o x y p t e r i d i n e-6-y l) m e t h y l] a m i n o\} b e n z o y l)-$ $N^{5}$-\{2-[(1,7-dicarba-closo-dodecaboran-1-yl)methyl-(1,2:3,4-di-O-isopropyliden-6-deoxy- $\alpha$ D-galactopyranos-6-yl)amino]ethyl\}-L-glutamine (8) or its isomer, and the disubstituted species, namely $(S)-2-(4-\{[(2-a m i n o-4-h y d r o x y p t e r i d i n e-6-y l) m e t h y l]$ amino $\}$ benzamido $)$ $N^{1}, N^{5}$-bis-\{2-[(1,7-dicarba-closo-dodecaboran-1-yl)methyl-(1,2:3,4-di-O-isopropylidene-6-de oxy- $\alpha$-D-galactopyranos-6-yl)amino]ethyl lamino)pentanediamide (9) (Scheme 3), also verified by high-resolution mass spectrometry $(m / z 883.5151$ for 8 and $m / z 1323.8829$ for 9). Obviously, in this case, the protocol from the literature [70] for conjugate formation with folic acid was not applicable, as we got a mixture of $\mathbf{8}$ and $\mathbf{9}$ in an unknown ratio. However, the obtained disubstituted folic acid derivative 9 has a high boron contents which could be useful for applications as a BNCT agent.

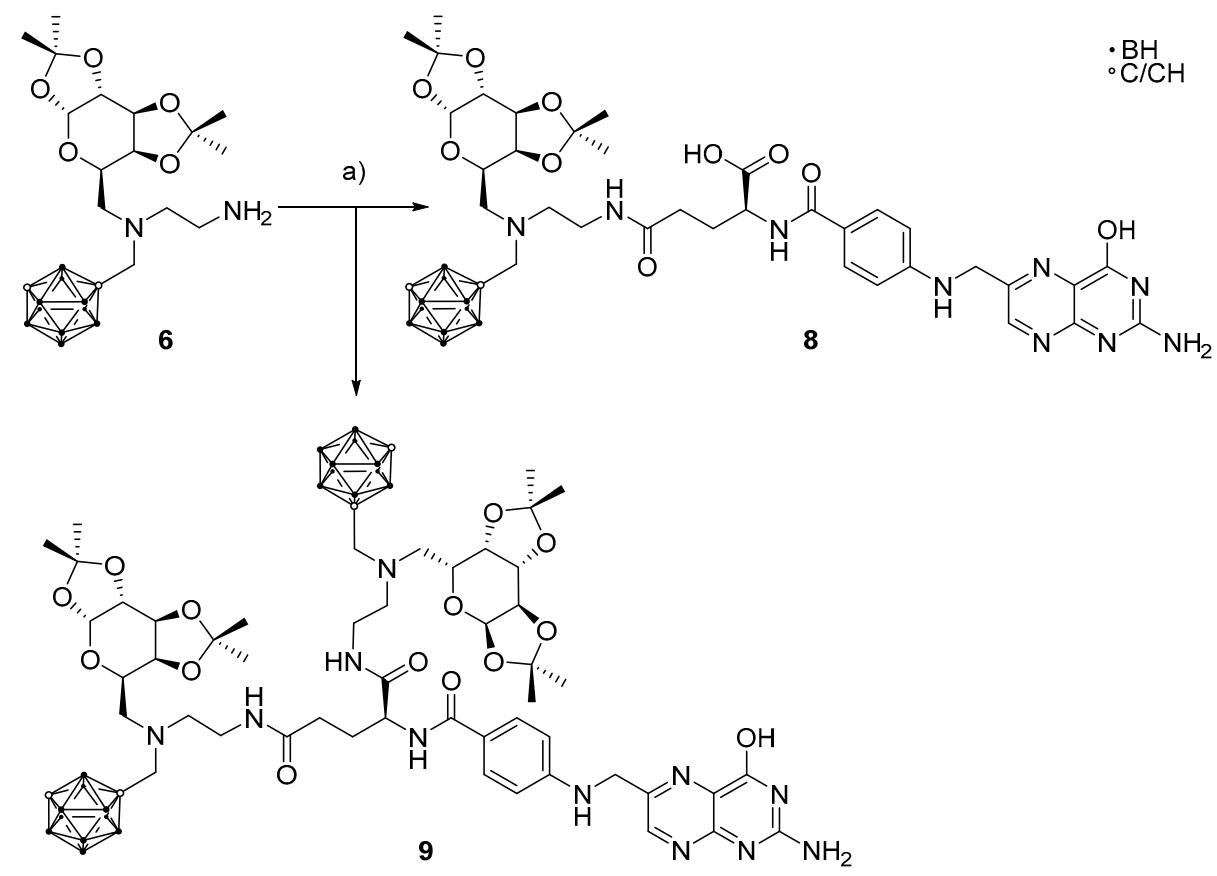

Scheme 3. (a) 1.00 eq. folic acid, 1.00 eq. DCC, 1.00 eq. NHS, 1.10 eq. DIPEA, dimethylformamide, no yield determined. 


\section{Materials and Methods}

All reactions were carried out under nitrogen atmosphere using Schlenk techniques, if not reported otherwise. Anhydrous diethyl ether and DCM were obtained with an MBRAUN solvent purification system MB SPS-800 (M. Braun Inertgas-Systeme GmbH, Garching, Germany). MeCN and 2,4,6-collidine were dried over calcium hydride and distilled prior to use. Anhydrous tetrahydrofuran was dried over potassium and distilled prior to use. All solvents were stored over a molecular sieve $(3 \AA)$ under nitrogen atmosphere. 1,2Dicarba-closo-dodecaborane(12) and 1,7-dicarba-closo-dodecaborane(12) are commercially available. 1-(Hydroxymethyl)-1,2-dicarba-closo-dodecaborane(12) [71], 1-(hydroxymethyl)1,7-dicarba-closo-dodecaborane(12) [57], 1-(trifluoromethanesulfonylmethyl)-1,2-dicarbacloso-dodecaborane(12) [58], 1-(trifluoromethanesulfonylmethyl)-1,7-dicarba-closo-dodecab orane(12) [58], 1,2:3,4-di-O-isopropylidene-6-deoxy- $\alpha$-D-galactopyranos-6-yltriflat [60] and 7-amino-4-methylcoumarin [72] were synthesized according to respective protocols from the literature. All other chemicals were commercially available and were used as received.

Thin-layer chromatography (TLC) with silica gel $60 \mathrm{~F}_{254}$ on glass, available from Merck KGaA (Darmstadt, Germany), or ALUGRAM ${ }^{\circledR}$ XTRA SIL G/UV ${ }_{254}$ from MachereyNagel GmbH \& Co. KG (Düren, Germany) on aluminum foil were used for monitoring the reactions. Carborane-containing spots were visualized with a $5 \%$ solution of $\mathrm{PdCl}_{2}$ in methanol. Non-carborane-containing spots were visualized with a basic potassium permanganate solution. For chromatography, silica gel $(60 \AA)$ with a particle diameter in the range of 0.035 to $0.070 \mathrm{~mm}$ was used. Prior to column chromatography, raw products were adsorbed on Celite ${ }^{\circledR} \mathrm{S}$ from Sigma-Aldrich Chemie GmbH (Taufkirchen, Germany).

NMR measurements were carried out on a Bruker AVANCE III HD spectrometer (Bruker Corporation, Billerica, MA, United States of America) with an Ascend ${ }^{\mathrm{TM}} 400$ magnet (Bruker Corporation, Billerica, MA, United States of America) at room temperature. Tetramethylsilane was used as internal standard for ${ }^{1} \mathrm{H}$ - and ${ }^{13} \mathrm{C}\left\{{ }^{1} \mathrm{H}\right\}-\mathrm{NMR}$ spectra; ${ }^{11} \mathrm{~B}$ and ${ }^{11} \mathrm{~B}\left\{{ }^{1} \mathrm{H}\right\}$-NMR spectra were referenced to the $\Xi$ scale [73]. NMR spectra were recorded at the following frequencies: ${ }^{1} \mathrm{H}: 400.16 \mathrm{MHz},{ }^{13} \mathrm{C}: 100.63 \mathrm{MHz},{ }^{11} \mathrm{~B}: 128.38 \mathrm{MHz}$. All chemical shifts are reported in parts per million (ppm). Assignment of the ${ }^{1} \mathrm{H}$ and ${ }^{13} \mathrm{C}$ signals was based on 2D-NMR spectra (H,H-COSY, H,C-HSQC, H,C-HMQC and H,C-HMBC). NMR data were interpreted with MestReNova [74]. NMR signals that appeared as broad overlapping signals with the shape of a multiplet or singlet in either ${ }^{1} \mathrm{H}-,{ }^{11} \mathrm{~B}\left\{{ }^{1} \mathrm{H}\right\}$ - or ${ }^{11} \mathrm{~B}$ NMR spectra were described as "br" (broad). The numbering scheme of the compounds for assignment of NMR signals is given in the Supplementary Materials (see Figure S1).

IR data were obtained with a PerkinElmer FT-IR spectrometer Spectrum 2000 (Perkin Elmer, Inc., Waltham, MA, United States of America) as KBr pellets and with a Thermo Scientific Nicolet iS5 with an ATR unit (Thermo Fisher Scientific, Waltham, MA, United States of America) in the range from 4000 to $400 \mathrm{~cm}^{-1}$.

High-resolution electrospray ionization mass spectrometry (ESI-HRMS) was performed with an ESI ESQUIRE 3000 PLUS spectrometer (Bruker Corporation, Billerica, MA, United States of America) with an IonTrap-analyzer from Bruker Daltonics or on a MicroTOF spectrometer from Bruker Daltonics with a ToF analyzer in negative or positive mode. As solvents for the measurements, $\mathrm{DCM}, \mathrm{MeCN}$, methanol or mixtures of these solvents were used. Interpretation of the spectra was carried out using MestReNova [74].

Tert-Butyl-N-(1,2:3,4-di-O-isopropylidene-6-deoxy- $\alpha$-D-galactopyranos-6-yl)glycinate (1): Diisopropylethylamine $(2.55 \mathrm{~mL}, 15.0 \mathrm{mmol}, 2.96 \mathrm{eq}$.) was added dropwise under nitrogen atmosphere at room temperature to a solution of tert-butyl glycinate hydrochloride (1.12 g, $6.12 \mathrm{mmol}, 1.20$ eq.) and 1,2:3,4-di-O-isopropylidene-6-deoxy- $\alpha$-D-galactopyranos6-trifluoromethanesulfonate $(2.00 \mathrm{~g}, 5.10 \mathrm{mmol}, 1.00$ eq.) in $50 \mathrm{~mL} \mathrm{MeCN}$. The reaction mixture was stirred for $72 \mathrm{~h}$ at $45^{\circ} \mathrm{C}$. The reaction was stopped by evaporating all volatile components under reduced pressure at $45^{\circ} \mathrm{C}$. The crude product was dissolved in ethyl acetate $(100 \mathrm{~mL})$ and washed with $\mathrm{H}_{2} \mathrm{O}$ and saturated $\mathrm{NaCl}$ solution. Subsequently, the aqueous layer was extracted with ethyl acetate $(3 \times 50 \mathrm{~mL})$, dried with $\mathrm{Na}_{2} \mathrm{SO}_{4}$, filtered and the solvent was removed under reduced pressure. The crude material was puri- 
fied by column chromatography using $n$-hexane/ethanol $\left(14: 1\left(R_{\mathrm{f}}=0.41\right)\right.$ to $10: 1\left(R_{\mathrm{f}}=\right.$ $0.58),(v / v))$ as eluent. Compound 1 was obtained as colorless oil in $68 \%$ yield (1.30 g, $3.48 \mathrm{mmol}) .{ }^{1} \mathrm{H}-\mathrm{NMR}\left(400 \mathrm{MHz}\right.$, chloroform- $\left.d_{1}\right): \delta[\mathrm{ppm}]=1.33,1.34,1.45$ and $1.54(\mathrm{~s}$, $12 \mathrm{H},{ }^{13}, 13^{\prime}, 14$ and $\left.14^{\prime} \mathrm{CH}_{3}\right), 1.46\left(\mathrm{~s}, 9 \mathrm{H}, 10,10^{\prime}\right.$ and $\left.10^{\prime \prime} \mathrm{CH}_{3}\right), 2.77$ to $2.92\left(\mathrm{~m}, 2 \mathrm{H},{ }^{6} \mathrm{CH}_{2}\right), 3.26$ to $3.40\left(\mathrm{~m}, 2 \mathrm{H},{ }^{7} \mathrm{CH}_{2}\right), 3.88\left(\mathrm{~m}, 1 \mathrm{H},{ }^{5} \mathrm{CH}\right), 4.22\left(\mathrm{dd}, 1 \mathrm{H},{ }^{4} \mathrm{CH},{ }^{3} \mathrm{~J}_{\mathrm{HH}}=7.9 \mathrm{~Hz},{ }^{3} \mathrm{~J}_{\mathrm{HH}}=4.9 \mathrm{~Hz}\right)$, $4.31\left(\mathrm{dd}, 1 \mathrm{H},{ }^{2} \mathrm{CH},{ }^{3} \mathrm{~J}_{\mathrm{HH}}=5.1 \mathrm{~Hz},{ }^{3} \mathrm{~J}_{\mathrm{HH}}=2.4 \mathrm{~Hz}\right), 4.60\left(\mathrm{dd}, 1 \mathrm{H},{ }^{3} \mathrm{CH},{ }^{3} J_{\mathrm{HH}}=7.9 \mathrm{~Hz},{ }^{3} J_{\mathrm{HH}}\right.$ $=4.9 \mathrm{~Hz}), 5.54\left(\mathrm{~d}, 1 \mathrm{H},{ }^{1} \mathrm{CH},{ }^{3} J_{\mathrm{HH}}=5.1 \mathrm{~Hz}\right) \cdot{ }^{13} \mathrm{C}\left\{{ }^{1} \mathrm{H}\right\}-\mathrm{NMR}\left(100 \mathrm{MHz}\right.$, chloroform- $\left.d_{1}\right): \delta$

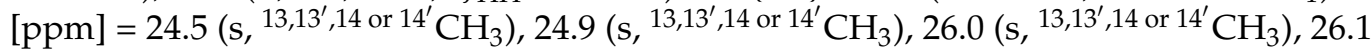
$\left(\mathrm{s}, 13,13^{\prime}, 14\right.$ or $\left.14^{\prime} \mathrm{CH}_{3}\right), 28.1\left(\mathrm{~s},{ }^{10,10^{\prime}}\right.$ and $\left.10^{\prime \prime} \mathrm{CH}_{3}\right), 49.1\left(\mathrm{~s},{ }^{6} \mathrm{CH}_{2}\right), 51.8\left(\mathrm{~s},{ }^{7} \mathrm{CH}_{2}\right), 67.1\left(\mathrm{~s},{ }^{5} \mathrm{CH}\right)$, $70.5\left(\mathrm{~s},{ }^{2} \mathrm{CH}\right), 70.8\left(\mathrm{~s},{ }^{3} \mathrm{CH}\right), 71.9\left(\mathrm{~s},{ }^{4} \mathrm{CH}\right), 80.9\left(\mathrm{~s},{ }^{9} \mathrm{C}_{\mathrm{q}}\right), 96.4\left(\mathrm{~s},{ }^{1} \mathrm{CH}\right), 108.4\left(\mathrm{~s},{ }^{11} \mathrm{C}_{\mathrm{q}}\right), 109.1(\mathrm{~s}$, $\left.{ }^{12} \mathrm{C}_{\mathrm{q}}\right), 171.4\left(\mathrm{~s},{ }^{8} \mathrm{C}_{\mathrm{q}}\right) . \mathrm{IR}(\mathrm{KBr}): \tilde{v}=2982\left(\mathrm{~m}, v \mathrm{CH}-\mathrm{sp}^{3}\right), 2935\left(\mathrm{~m}, v \mathrm{CH}-\mathrm{sp}^{3}\right), 2361(\mathrm{w}), 1736(\mathrm{~s}$, $\left.v \mathrm{CO}_{\text {Ester }}\right), 1459\left(\mathrm{w}, \delta_{\text {as. }} \mathrm{CH}_{2}\right), 1372\left(\mathrm{~m}, \delta_{\mathrm{s} .} \mathrm{CH}_{3}\right), 1256(\mathrm{~m}), 1213(\mathrm{~s}), 1165$ (s, vas. C-O-C Ester $)$, $1114(\mathrm{~m}), 1070$ (s, vC-O-C $\mathrm{Ether}), 1003(\mathrm{~m}), 918(\mathrm{w}), 898(\mathrm{w}), 854(\mathrm{w}), 804(\mathrm{w}), 771(\mathrm{w}), 650$ $(\mathrm{w}), 512(\mathrm{w}) \mathrm{cm}^{-1}$. ESI-HRMS: $(\mathrm{m} / z)$ calculated for $\left[\mathrm{NaC}_{18} \mathrm{H}_{31} \mathrm{NO}_{7}\right]^{+}=396.19986$; observed 396.20153 $\left[\mathrm{M}+\mathrm{Na}^{+}\right]^{+}$; calculated for $\left[\mathrm{C}_{18} \mathrm{H}_{32} \mathrm{NO}_{7}\right]^{+}=374.21791$; observed 374.21969 $[\mathrm{M}+\mathrm{H}]^{+}$; calculated for $\left[\mathrm{NaC}_{14} \mathrm{H}_{23} \mathrm{NO}_{7}\right]^{+}=340.13726$; observed 340.13844 [M-C $\left.\mathrm{H}_{8}+\mathrm{Na}\right]^{+}$; calculated for $\left[\mathrm{C}_{14} \mathrm{H}_{24} \mathrm{NO}_{7}\right]^{+}=318.15531$; observed $318.15632\left[\mathrm{M}-\mathrm{C}_{4} \mathrm{H}_{8}+\mathrm{H}\right]^{+}$.

Tert-Butyl-\{2-[(1,2:3,4-di-O-isopropylidene-6-deoxy- $\alpha$-D-galactopyranos-6-yl)amino]-ethyl $\}_{C a}$ rbamate (2): A $250 \mathrm{~mL}$ round-bottom flask was charged with $3.20 \mathrm{~g}$ (8.16 mmol, 0.76 eq.) 1,2:3,4-di-O-isopropylidene-6-deoxy- $\alpha$-D-galactopyranos-6-trifluormethanesulfonate and $60 \mathrm{~mL}$ MeCN. Then, $1.70 \mathrm{~mL}$ (1.73 g, $10.8 \mathrm{mmol}, 1.00 \mathrm{eq}$.) tert-butyl- $N$-(2-aminoethyl)carbamate, dissolved in $10 \mathrm{~mL} \mathrm{MeCN}$, were added via a dropping funnel to this solution. The reaction mixture was cooled to $0{ }^{\circ} \mathrm{C}$ and, subsequently, $2.05 \mathrm{~mL}(1.56 \mathrm{~g}, 12.1 \mathrm{mmol}, 1.12 \mathrm{eq}.) \mathrm{N}, \mathrm{N}$ diisopropylethylamine, dissolved in $10 \mathrm{~mL} \mathrm{MeCN}$, were added dropwise. After stirring for $15 \mathrm{~min}$ at $0{ }^{\circ} \mathrm{C}$, the reaction mixture was warmed to $40^{\circ} \mathrm{C}$ and stirred for two days. The reaction was stopped by cooling to room temperature and adding $30 \mathrm{~mL}$ saturated $\mathrm{NH}_{4} \mathrm{Cl}$ solution. All volatile components were removed under reduced pressure, and the remaining aqueous layer was extracted four times with $40 \mathrm{~mL}$ ethyl acetate, respectively. The combined organic layers were dried over $\mathrm{MgSO}_{4}$. The drying agent was filtered off and the solvent was removed under reduced pressure. The crude product was purified by column chromatography (a: DCM/methanol, 20:1, (v/v); b: acetone; c: $n$-hexane/isopropanol, $8: 1$ to $3: 1,(v / v))$. A total of $0.56 \mathrm{~g}$ tert-butyl-(2-\{[bis(1,2:3,4-di-O-isopropylidene-6-deoxy- $\alpha$ D-galactopyranos-6-yl)] amino\}ethyl)-carbamate $\left(2^{\prime}\right)\left(R_{\mathrm{f}}=0.38, \mathrm{DCM} /\right.$ methanol, 20:1, $(v / v)$, $0.87 \mathrm{mmol}, 8 \%$, analytical data are given in the Supplementary Materials) was obtained as a colorless solid, as well as $3.28 \mathrm{~g}$ tert-butyl-\{2-[(1,2:3,4-di-O-isopropylidene-6-deoxy$\alpha$-D-galactopyranos-6-yl)amino]ethyl $\}$ carbamate $(2)\left(R_{\mathrm{f}}=0.12 n\right.$-hexane/isopropanol, 5:1, $(v / v), 8.27 \mathrm{mmol}, 77 \%)$ as a colorless solid. ${ }^{1} \mathrm{H}-\mathrm{NMR}\left(400 \mathrm{MHz}\right.$, acetone- $\left.d_{6}\right): \delta[\mathrm{ppm}]=$

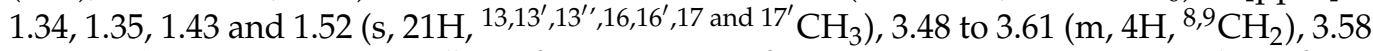
$\left(\mathrm{m}, 2 \mathrm{H},{ }^{6} \mathrm{CH}_{2}\right), 4.28\left(\mathrm{td}, 1 \mathrm{H},{ }^{5} \mathrm{CH},{ }^{3} \mathrm{~J}_{\mathrm{HH}}=6.0 \mathrm{~Hz},{ }^{3} \mathrm{~J}_{\mathrm{HH}}=1.8 \mathrm{~Hz}\right), 4.39\left(\mathrm{dd}, 1 \mathrm{H},{ }^{4} \mathrm{CH},{ }^{3} J_{\mathrm{HH}}\right.$ $\left.=7.9 \mathrm{~Hz},{ }^{3} J_{\mathrm{HH}}=1.9 \mathrm{~Hz}\right), 4.45\left(\mathrm{dd}, 1 \mathrm{H},{ }^{2} \mathrm{CH},{ }^{3} J_{\mathrm{HH}}=5.0 \mathrm{~Hz}^{3} J_{\mathrm{HH}}=2.5 \mathrm{~Hz}\right), 4.73(\mathrm{dd}, 1 \mathrm{H}$, $\left.{ }^{3} \mathrm{CH},{ }^{3} J_{\mathrm{HH}}=7.8 \mathrm{~Hz},{ }^{3} \mathrm{~J}_{\mathrm{HH}}=2.5 \mathrm{~Hz}\right), 5.56\left(\mathrm{~d}, 1 \mathrm{H},{ }^{1} \mathrm{CH},{ }^{3} \mathrm{~J}_{\mathrm{HH}}=4.9 \mathrm{~Hz}\right), 6.64\left(\mathrm{~s}, 1 \mathrm{H},{ }^{7} \mathrm{NH}\right)$, $8.24\left(\mathrm{~s}, 1 \mathrm{H},{ }^{10} \mathrm{NH}\right) .{ }^{13} \mathrm{C}\left\{{ }^{1} \mathrm{H}\right\}-\mathrm{NMR}\left(100 \mathrm{MHz}\right.$, acetone- $\left.d_{6}\right): \delta[\mathrm{ppm}]=24.4,25.0,26.2,28.5$ $\left(\mathrm{s},{ }^{13}, 13^{\prime}, 13^{\prime \prime} 16,16^{\prime}, 17\right.$ and $\left.17^{\prime} \mathrm{CH}_{3}\right), 38.2\left(\mathrm{~s},{ }^{6} \mathrm{CH}_{2}\right), 49.3\left(\mathrm{~s},{ }^{8}\right.$ or $\left.{ }^{9} \mathrm{CH}_{2}\right), 50.7\left(\mathrm{~s},{ }^{8}\right.$ or $\left.{ }^{9} \mathrm{CH}_{2}\right), 64.6$ (s, $\left.{ }^{5} \mathrm{CH}\right), 71.2\left(\mathrm{~s},{ }^{2} \mathrm{CH}\right), 71.6\left(\mathrm{~s},{ }^{3} \mathrm{CH}\right), 72.2\left(\mathrm{~s},{ }^{4} \mathrm{CH}\right), 80,6\left(\mathrm{~s},{ }^{12} \mathrm{C}_{\mathrm{q}}\right), 97.1\left(\mathrm{~s},{ }^{1} \mathrm{CH}\right) 109.9$ and 110.7 $\left(\mathrm{s},{ }^{14},{ }^{15} \mathrm{C}_{\mathrm{q}}\right)$. Carbonyl carbon atom ${ }^{11} \mathrm{C}$ was not observed.

Tert-Butyl-N-[(1,7-dicarba-closo-dodecaboran-1-yl)methyl]-N-(1,2:3,4-di-O-isopropylidene-6deoxy- $\alpha$-D-galacatopyranos-6-yl)glycinate (3): A suspension of tert-butyl-N-(1,2:3,4-di-Oisopropylidene-6-deoxy- $\alpha$-D-galactopyranos-6-yl)glycinate (1) (0.94 g, $2.52 \mathrm{mmol}, 1.00$ eq.), 1-(trifluoromethanesulfonylmethyl)-1,7-dicarba-closo-dodecaborane (0.93 g, $3.02 \mathrm{mmol}$, 1.20 eq.) and potassium carbonate $(0.42 \mathrm{~g}, 3.02 \mathrm{mmol}, 1.20$ eq. $)$ in $10 \mathrm{~mL}$ toluene was stirred at $95^{\circ} \mathrm{C}$ for $43 \mathrm{~h}$ under nitrogen atmosphere. Then, the suspension was diluted with ethyl acetate and washed with $20 \mathrm{~mL} \mathrm{H}_{2} \mathrm{O}$ and saturated $\mathrm{NaCl}$ solution. Subsequently, the aqueous layer was extracted with ethyl acetate $(3 \times 15 \mathrm{~mL})$, the combined organic layers were dried over $\mathrm{MgSO}_{4}$ and filtered, and the solvent was removed under reduced pres- 
sure. The crude material was purified by column chromatography using $n$-hexane/ethyl acetate $\left(20: 1,(v / v), R_{\mathrm{f}}=0.50\right)$ and then $n$-hexane/ethanol $\left(20: 1,(v / v), R_{\mathrm{f}}=0.27\right)$ as eluent. Compound 3 was obtained as a colorless oil in $54 \%$ yield $(721 \mathrm{mg}, 1.36 \mathrm{mmol}) .{ }^{1} \mathrm{H}-\mathrm{NMR}$ $\left(400 \mathrm{MHz}\right.$, chloroform- $\left.d_{1}\right): \delta[\mathrm{ppm}]=1.34,1.42$ and $1.56\left(\mathrm{~s}, 12 \mathrm{H}, 13,13^{\prime}, 14\right.$ and $\left.14^{\prime} \mathrm{CH}_{3}\right), 1.44(\mathrm{~s}$,

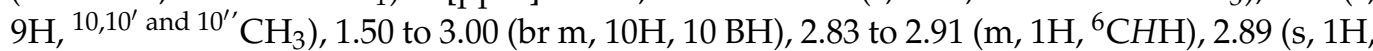
$\left.{ }^{17} \mathrm{CH}\right), 2.97$ to $3.09\left(\mathrm{dd}, 1 \mathrm{H},{ }^{6} \mathrm{CHH},{ }^{2} J_{\mathrm{HH}}=14.1 \mathrm{~Hz},{ }^{3} \mathrm{~J}_{\mathrm{HH}}=7.0 \mathrm{~Hz}\right), 3.13\left(\mathrm{~d}, 1 \mathrm{H},{ }^{15} \mathrm{CHH},{ }^{2} J_{\mathrm{HH}}\right.$ $=15.7 \mathrm{~Hz}), 3.23\left(\mathrm{~d}, 1 \mathrm{H},{ }^{15} \mathrm{CHH},{ }^{3} \mathrm{~J}_{\mathrm{HH}}=15.7 \mathrm{~Hz}\right), 3.36\left(\mathrm{~d}, 1 \mathrm{H},{ }^{7} \mathrm{CHH},{ }^{3} J_{\mathrm{HH}}=17.8 \mathrm{~Hz}\right), 3.53$ $\left(\mathrm{d}, 1 \mathrm{H},{ }^{7} \mathrm{CHH},{ }^{3} J_{\mathrm{HH}}=17.8 \mathrm{~Hz}\right), 3.84\left(\mathrm{~m}, 1 \mathrm{H},{ }^{5} \mathrm{CH}\right), 4.25\left(\mathrm{dd}, 1 \mathrm{H},{ }^{4} \mathrm{CH},{ }^{3} J_{\mathrm{HH}}=7.9 \mathrm{~Hz},{ }^{3} J_{\mathrm{HH}}=\right.$ $1.9 \mathrm{~Hz}), 4.29\left(\mathrm{dd}, 1 \mathrm{H},{ }^{2} \mathrm{CH},{ }^{3} \mathrm{~J}_{\mathrm{HH}}=5.1 \mathrm{~Hz},{ }^{3} J_{\mathrm{HH}}=2.4 \mathrm{~Hz}\right), 4.60\left(\mathrm{dd}, 1 \mathrm{H},{ }^{3} \mathrm{CH},{ }^{3} J_{\mathrm{HH}}=7.9 \mathrm{~Hz}\right.$, $\left.{ }^{3} J_{\mathrm{HH}}=2.4 \mathrm{~Hz}\right), 5.50\left(\mathrm{~d}, 1 \mathrm{H},{ }^{1} \mathrm{CH},{ }^{3} J_{\mathrm{HH}}=5.1 \mathrm{~Hz}\right) .{ }^{13} \mathrm{C}\left\{{ }^{1} \mathrm{H}\right\}-\mathrm{NMR}\left(100 \mathrm{MHz}\right.$, chloroform- $\left.d_{1}\right)$ :

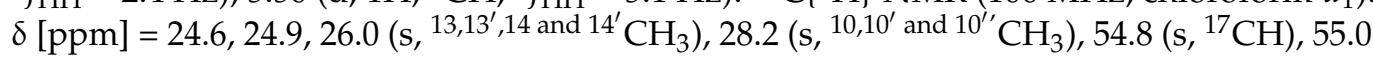
$\left(\mathrm{s},{ }^{6} \mathrm{CH}_{2}\right), 55.9\left(\mathrm{~s},{ }^{7} \mathrm{CH}_{2}\right), 60.9\left(\mathrm{~s},{ }^{15} \mathrm{CH}_{2}\right), 66.8\left(\mathrm{~s},{ }^{5} \mathrm{CH}\right), 70.4\left(\mathrm{~s},{ }^{2} \mathrm{CH}\right), 70.8\left(\mathrm{~s},{ }^{3} \mathrm{CH}\right), 71.9$ $\left(\mathrm{s},{ }^{4} \mathrm{CH}\right), 78.5\left(\mathrm{~s},{ }^{16} \mathrm{C}_{\mathrm{q}}\right), 81.5\left(\mathrm{~s},{ }^{9} \mathrm{C}_{\mathrm{q}}\right), 96.5\left(\mathrm{~s},{ }^{1} \mathrm{CH}\right), 108.5$ and $109.1\left(\mathrm{~s},{ }^{11,12} \mathrm{C}_{\mathrm{q}}\right), 170.8(\mathrm{~s}$, $\left.{ }^{8} \mathrm{C}_{\mathrm{q}}\right) .{ }^{11} \mathrm{~B}\left\{{ }^{1} \mathrm{H}\right\}-\mathrm{NMR}\left(128 \mathrm{MHz}\right.$, chloroform- $\left.d_{1}\right): \delta[\mathrm{ppm}]=-15.7(\mathrm{~s}, 2 \mathrm{~B}),-13.6(\mathrm{~s}, 2 \mathrm{~B}),-10.9$ $(\mathrm{s}, 4 \mathrm{~B}),-9.5(\mathrm{~s}, 1 \mathrm{~B}),-4.4(\mathrm{~s}, 1 \mathrm{~B})$. ESI-HRMS: $(\mathrm{m} / z)$ calculated for $\left[\mathrm{NaC}_{21} \mathrm{H}_{43} \mathrm{~B}_{10} \mathrm{NO}_{7}\right]^{+}=$ 552.3941; observed $552.3966[\mathrm{M}+\mathrm{Na}]^{+}$.

Tert-Butyl-\{2-[(1,7-dicarba-closo-dodecaboran-1-yl)methyl-(1,2:3,4-di-O-isopropyliden-6-deox

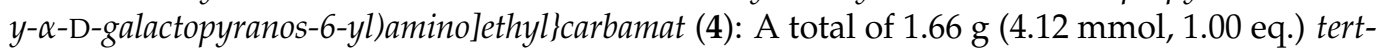
butyl-\{2-[(1,2:3,4-di-O-isopropylidene-6-deoxy- $\alpha$-D-galactopyranos-6-yl)amino]-ethyl $\}$ carba mate (2) was placed in a $50 \mathrm{~mL}$ round-bottom flask and dissolved in $10 \mathrm{~mL}$ toluene. Subsequently, $1.51 \mathrm{~g}$ (4.94 mmol, 1.20 eq.) 1-(trifluoromethanesulfonylmethyl)-1,7-dicarba-closododecaborane(12), dissolved in $10 \mathrm{~mL}$ toluene, and $0.68 \mathrm{~g}$ (4.94 mmol, 1.20 eq.) $\mathrm{K}_{2} \mathrm{CO}_{3}$ were added. The suspension was heated to $98{ }^{\circ} \mathrm{C}$ and stirred for $48 \mathrm{~h}$. The reaction was stopped by adding $20 \mathrm{~mL}$ distilled water and $4 \mathrm{~mL}$ saturated $\mathrm{NaCl}$ solution. The aqueous layer and organic layer were separated. The aqueous layer was extracted two times with $20 \mathrm{~mL}$ ethyl acetate. The combined organic layers were dried over $\mathrm{MgSO}_{4}$, the drying agent was filtered off and the solvent was removed under reduced pressure. The resulting yellow-brownish oil was purified by column chromatography (n-hexane/ethyl acetate, $5: 1,(v / v))$ and $4\left(1.17 \mathrm{~g}, 2.09 \mathrm{mmol}, 51 \%, R_{\mathrm{f}}=0.24\right.$ (n-hexane/ethyl acetate, 5:1, $\left.\left.(v / v)\right)\right)$ was isolated as a colorless foamy solid. ${ }^{1} \mathrm{H}-\mathrm{NMR}\left(400 \mathrm{MHz}\right.$, chloroform- $\left.d_{1}\right): \delta[\mathrm{ppm}]=1.19$ to $3.41(\mathrm{~m}, \mathrm{br}, 10 \mathrm{H}, 10 \mathrm{BH}), 1.34,1.36,1.45$ and $1.56\left(\mathrm{~s}, 12 \mathrm{H}, 15,15^{\prime}, 16\right.$ and $\left.16^{\prime} \mathrm{CH}_{3}\right), 1.45(\mathrm{~s}, 9 \mathrm{H}$, $12,12^{\prime}$ and $\left.12^{\prime \prime} \mathrm{CH}_{3}\right), 2.55$ to $2.63\left(\mathrm{~m}, 1 \mathrm{H},{ }^{7}\right.$ or $\left.{ }^{8} \mathrm{CHH}\right), 2.63$ to $2.72\left(\mathrm{~m}, 1 \mathrm{H},{ }^{6} \mathrm{CHH}\right), 2.80$ to 2.88 $\left(\mathrm{m}, 2 \mathrm{H},{ }^{7}\right.$ or ${ }^{8} \mathrm{CHH}$ and $\left.{ }^{6} \mathrm{CHH}\right), 2.90\left(\mathrm{~s}, 1 \mathrm{H},{ }^{19} \mathrm{CH}\right), 2.94$ to $3.07\left(\mathrm{~m}, 2 \mathrm{H},{ }^{17} \mathrm{CHH}\right.$ and $\left.{ }^{17} \mathrm{CHH}\right)$, 3.10 to $3.25\left(\mathrm{~m}, 2 \mathrm{H},{ }^{7}\right.$ and $\left.{ }^{8} \mathrm{CHH}\right), 3.85\left(\mathrm{~m}, 1 \mathrm{H},{ }^{5} \mathrm{CH}\right), 4.24\left(\mathrm{~d}, 1 \mathrm{H},{ }^{4} \mathrm{CH},{ }^{3} \mathrm{~J}_{\mathrm{HH}}=7.9 \mathrm{~Hz}\right), 4.31$ $\left(\mathrm{dd}, 1 \mathrm{H},{ }^{2} \mathrm{CH},{ }^{3} J_{\mathrm{HH}}=5.1 \mathrm{~Hz},{ }^{3} \mathrm{~J}_{\mathrm{HH}}=2.4 \mathrm{~Hz}\right), 4.62\left(\mathrm{dd}, 1 \mathrm{H},{ }^{3} \mathrm{CH},{ }^{3} \mathrm{~J}_{\mathrm{HH}}=7.9 \mathrm{~Hz},{ }^{3} J_{\mathrm{HH}}=\right.$ $2.4 \mathrm{~Hz}), 5.35\left(\mathrm{~s}, \mathrm{br}, 1 \mathrm{H},{ }^{9} \mathrm{NH}\right), 5.51\left(\mathrm{~d}, 1 \mathrm{H},{ }^{1} \mathrm{CH},{ }^{3} J_{\mathrm{HH}}=5.1 \mathrm{~Hz}\right) .{ }^{13} \mathrm{C}\left\{{ }^{1} \mathrm{H}\right\}-\mathrm{NMR}(100 \mathrm{MHz}$, chloroform- $\left.d_{1}\right): \delta[\mathrm{ppm}]=24.4,24.9,26.0\left(\mathrm{~s}, 15,15^{\prime}, 16\right.$ and $\left.16^{\prime} \mathrm{CH}_{3}\right), 28.5\left(\mathrm{~s}, 12,12^{\prime}\right.$ and $\left.12^{\prime \prime} \mathrm{CH}_{3}\right)$, $38.4\left(\mathrm{~s},{ }^{7}\right.$ or $\left.{ }^{8} \mathrm{CH}_{2}\right), 53.0\left(\mathrm{~s},{ }^{6} \mathrm{CH}_{2}\right), 54.6\left(\mathrm{~s},{ }^{7}\right.$ or $\left.{ }^{8} \mathrm{CH}_{2}\right) 54.7\left(\mathrm{~s},{ }^{19} \mathrm{CH}\right), 60.7\left(\mathrm{~s},{ }^{17} \mathrm{CH}_{2}\right), 65.8(\mathrm{~s}$, $\left.{ }^{5} \mathrm{CH}\right), 70.4\left(\mathrm{~s},{ }^{2} \mathrm{CH}\right), 70.9\left(\mathrm{~s},{ }^{3} \mathrm{CH}\right), 71.8\left(\mathrm{~s},{ }^{4} \mathrm{CH}\right), 77.8\left(\mathrm{~s},{ }^{18} \mathrm{C}_{\mathrm{q}}\right), 79.0\left(\mathrm{~s},{ }^{11} \mathrm{C}_{\mathrm{q}}\right), 96.5\left(\mathrm{~s},{ }^{1} \mathrm{CH}\right)$, 108.4 and $109.3\left(\mathrm{~s},{ }^{13}\right.$ and $\left.{ }^{14} \mathrm{C}_{\mathrm{q}}\right), 156.1\left(\mathrm{~s},{ }^{10} \mathrm{C}_{\mathrm{q}}\right) .{ }^{11} \mathrm{~B}\left\{{ }^{1} \mathrm{H}\right\}-\mathrm{NMR}\left(128 \mathrm{MHz}\right.$, chloroform- $\left.d_{1}\right): \delta$ [ppm] $=-15.6(\mathrm{~s}, 2 \mathrm{~B}),-13.6(\mathrm{~s}, 2 \mathrm{~B}),-10.8(\mathrm{~s}, 4 \mathrm{~B}),-9.3(\mathrm{~s}, 1 \mathrm{~B}),-4.3(\mathrm{~s}, 1 \mathrm{~B})$. IR $(\mathrm{KBr}): \tilde{v}=$ $3390(\mathrm{w}, v \mathrm{NH}), 2979\left(\mathrm{w}, v \mathrm{CH}-\mathrm{sp}^{3}\right), 2933\left(\mathrm{w}, v \mathrm{CH}-\mathrm{sp}^{3}\right), 2594(\mathrm{~m}, v B H), 1707(\mathrm{~m}$, amide I), $1504\left(\mathrm{~m}\right.$, amide II), $1455(\mathrm{~m}, \delta \mathrm{CH}), 1366(\mathrm{~m}, \delta \mathrm{CH}), 1252\left(\mathrm{~m}\right.$, amide III) $\mathrm{cm}^{-1}$. ESI-HRMS: $(m / z)$ calculated for $\left[\mathrm{C}_{22} \mathrm{H}_{47} \mathrm{~B}_{10} \mathrm{~N}_{2} \mathrm{O}_{7}\right]^{+}=559.4386$; observed $559.4395[\mathrm{M}+\mathrm{H}]^{+}$; calculated for $\left[\mathrm{NaC}_{22} \mathrm{H}_{46} \mathrm{~B}_{10} \mathrm{~N}_{2} \mathrm{O}_{7}\right]^{+}=581.4282$; observed $581.4208[\mathrm{M}+\mathrm{Na}]^{+}$.

$N-[(1,7-d i c a r b a-c l o s o-d o d e c a b o r a n e-1-y l) m e t h y l]-N-(1,2: 3,4-d i-O-i s o p r o p y l i d e n e-6-d e o x y-\alpha-D-$ galacatopyranos-6-yl)glycine (5): Method A. Anhydrous TFA (4.20 mL, $54.4 \mathrm{mmol}, 40.0$ eq.) was added dropwise under nitrogen atmosphere at $0{ }^{\circ} \mathrm{C}$ to a solution of tert-butyl- $N-[(1,7-$ dicarba-closo-dodecaboran-1-yl)methyl]-N-(1,2:3,4-di-O-isopropylidene-6-deoxy- $\alpha$-D-galaca topyranos-6-yl)glycinate (3) (0.71 g, $1.36 \mathrm{mmol}, 1.00 \mathrm{eq}$.) in $4.20 \mathrm{~mL}$ DCM. The mixture was warmed to room temperature and stirred for $3 \mathrm{~h}$. When the reaction was completed, TFA and DCM were removed under reduced pressure. DCM and diethyl ether were used as an entrainer to remove remaining TFA. The crude material was purified by column chromatography using $n$-hexane/ethyl acetate $(10: 1,(v / v))$ as eluent. Diethyl ether was 
used to remove remaining solvent. Compound $\mathbf{5}$ was obtained as a colorless solid in $47 \%$ yield (300 mg, $0.63 \mathrm{mmol})$.

Method B. A Schlenk flask was charged with tert-butyl-N-[(1,7-dicarba-closo-dodecabor an-1-yl)methyl]-N-(1,2:3,4-di-O-isopropylidene-6-deoxy- $\alpha$-D-galacatopyranos-6-yl)-glycina te (3) $(0.50 \mathrm{~g}, 0.94 \mathrm{mmol}, 1.00 \mathrm{eq}$.). Then, $4.0 \mathrm{~mL}$ anhydrous TFA (52.2 mmol, 55.6 eq.) were added and the resulting solution was stirred at room temperature for $3 \mathrm{~h}$. Afterwards, about $4 \mathrm{~mL}$ DCM were added and both, TFA and DCM, were removed under reduced pressure. This process was repeated three more times with DCM as an entrainer to remove remaining TFA. Then, $3 \mathrm{~mL}$ concentrated $\mathrm{NaHCO}_{3}$ solution were added to the obtained crude product while stirring and the mixture was subsequently sonicated for $15 \mathrm{~min}$, resulting in a thick, cloudy white suspension with a brownish oil-like layer on top. Upon addition of $2 \mathrm{~mL}$ DCM, the oil-like layer was dissolved and gas evolution was observed. Once the gas evolution had almost stopped, the solution was stirred for five more minutes. Subsequently, the aqueous layer was removed using a syringe and extracted two times with $2 \mathrm{~mL}$ ethyl acetate each. The combined organic layers were washed twice with $2 \mathrm{~mL}$ of water. Afterwards, the combined organic layers were dried over $\mathrm{MgSO}_{4}$ and filtered. Finally, the solvent was removed under reduced pressure, affording compound 5 as a yellowish foamy solid in 65\% yield (290 mg, $0.612 \mathrm{mmol}) .{ }^{1} \mathrm{H}-\mathrm{NMR}$ (400 MHz, chloroform$\left.d_{1}\right): \delta[\mathrm{ppm}]=1.33,1.34,1.43$ and $1.56\left(\mathrm{~s}, 12 \mathrm{H}, 11,11^{\prime}, 12\right.$ and $\left.12^{\prime} \mathrm{CH}_{3}\right), 1.50$ to 3.00 (m, br, $10 \mathrm{H}$, $10 \mathrm{BH}), 2.91$ to $2.99\left(\mathrm{~m}, 3 \mathrm{H},{ }^{6} \mathrm{CH}_{2}\right.$ and $\left.{ }^{15} \mathrm{CH}\right), 3.17\left(\mathrm{~d}, 1 \mathrm{H},{ }^{13} \mathrm{CHH},{ }^{2} J_{\mathrm{HH}}=20.0 \mathrm{~Hz}\right), 3.21(\mathrm{~d}$, $\left.1 \mathrm{H},{ }^{13} \mathrm{CHH},{ }^{2} J_{\mathrm{HH}}=20.0 \mathrm{~Hz}\right), 3.48\left(\mathrm{~d}, 1 \mathrm{H},{ }^{7} \mathrm{CHH},{ }^{2} J_{\mathrm{HH}}=18.2 \mathrm{~Hz}\right), 3.59\left(\mathrm{~d}, 1 \mathrm{H},{ }^{7} \mathrm{CHH},{ }^{2} J_{\mathrm{HH}}\right.$ $=18.2 \mathrm{~Hz}), 3.87\left(\mathrm{~m}, 1 \mathrm{H},{ }^{5} \mathrm{CH}\right), 4.15\left(\mathrm{dd}, 1 \mathrm{H},{ }^{4} \mathrm{CH},{ }^{3} J_{\mathrm{HH}}=7.9 \mathrm{~Hz},{ }^{3} J_{\mathrm{HH}}=2.0 \mathrm{~Hz}\right), 4.35(\mathrm{dd}$, $\left.1 \mathrm{H},{ }^{2} \mathrm{CH},{ }^{3} \mathrm{~J}_{\mathrm{HH}}=5.2 \mathrm{~Hz},{ }^{3} \mathrm{~J}_{\mathrm{HH}}=2,4 \mathrm{~Hz}\right), 4.62\left(\mathrm{dd}, 1 \mathrm{H},{ }^{3} \mathrm{CH},{ }^{3} J_{\mathrm{HH}}=7.9 \mathrm{~Hz},{ }^{3} \mathrm{~J}_{\mathrm{HH}}=2.4 \mathrm{~Hz}\right)$, $5.55\left(\mathrm{~d}, 1 \mathrm{H},{ }^{1} \mathrm{CH},{ }^{3} \mathrm{~J}_{\mathrm{HH}}=5.1 \mathrm{~Hz}\right) .{ }^{13} \mathrm{C}\left\{{ }^{1} \mathrm{H}\right\}-\mathrm{NMR}\left(100 \mathrm{MHz}\right.$, chloroform- $\left.d_{1}\right): \delta[\mathrm{ppm}]=24.4$,

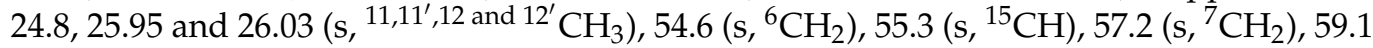
$\left(\mathrm{s},{ }^{13} \mathrm{CH}_{2}\right), 65.5\left(\mathrm{~s},{ }^{5} \mathrm{CH}\right), 70.4\left(\mathrm{~s},{ }^{2} \mathrm{CH}\right), 70.8\left(\mathrm{~s},{ }^{3} \mathrm{CH}\right), 71.6\left(\mathrm{~s},{ }^{4} \mathrm{CH}\right), 76.3\left(\mathrm{~s},{ }^{14} \mathrm{C}_{\mathrm{q}}\right), 96.4(\mathrm{~s}$, $\left.{ }^{1} \mathrm{CH}\right), 108.8$ and $109.7\left(\mathrm{~s},{ }^{9}\right.$ and $\left.{ }^{10} \mathrm{C}_{\mathrm{q}}\right), 172.4\left(\mathrm{~s},{ }^{8} \mathrm{C}_{\mathrm{q}}\right) .{ }^{11} \mathrm{~B}\left\{{ }^{1} \mathrm{H}\right\}-\mathrm{NMR}\left(128 \mathrm{MHz}\right.$, chloroform- $\left.d_{1}\right)$ : $\delta[\mathrm{ppm}]=-15.7(\mathrm{~s}, 2 \mathrm{~B}),-13.5(\mathrm{~s}, 2 \mathrm{~B}),-11.3(\mathrm{~s}, 2 \mathrm{~B}),-10.6(\mathrm{~s}, 2 \mathrm{~B}),-9.0(\mathrm{~s}, 1 \mathrm{~B}),-4.5(\mathrm{~s}, 1 \mathrm{~B})$. IR (KBr): $\tilde{v}=3061\left(\mathrm{w}, v \mathrm{CH}_{2}-\mathrm{sp}^{2}\right), 2985\left(\mathrm{w}, \mathrm{v}_{\mathrm{as}} . \mathrm{CH}_{2}-\mathrm{sp}^{3}\right), 2961\left(\mathrm{w}, \mathrm{v}_{\mathrm{s} .} \mathrm{CH}_{2}-\mathrm{sp}^{3}\right), 2931(\mathrm{~m}$, $\left.v_{\text {as. }} \mathrm{CH}_{3}-\mathrm{sp}^{3}\right), 2871\left(\mathrm{w}, v_{\mathrm{s}} \mathrm{CH}_{3}-\mathrm{sp}^{3}\right), 2595\left(\mathrm{~s}, v \mathrm{BH}-\mathrm{sp}^{3}\right), 1716\left(\mathrm{~m}, v \mathrm{C}=\mathrm{O}_{\text {carboxylic acid }}\right), 1456(\mathrm{~m}$, $\left.\delta_{\text {as. }} \mathrm{CH}_{3}-\mathrm{sp}^{3}\right), 13\left(\mathrm{~m}, \delta_{\mathrm{s} .} \mathrm{CH}_{3}-\mathrm{sp}^{3}\right), 1066\left(\mathrm{~s}, v \mathrm{C}-\mathrm{O}-\mathrm{C}_{\text {ether }}\right) \mathrm{cm}^{-1}$. ESI-HRMS: $(\mathrm{m} / \mathrm{z})$ calculated for $\left[\mathrm{C}_{17} \mathrm{H}_{36} \mathrm{~B}_{10} \mathrm{NO}_{7}\right]^{+}=474.3495$; observed $474.3485[\mathrm{M}+\mathrm{H}]^{+}$.

$N^{1}$-[(1,7-Dicarba-closo-dodecaborane-1-yl)methyl]-N $N^{1}-(1,2: 3,4-d i-O-i s o p r o p y l i d e n e-6-d e o x y-$ $\alpha$-D-galactopyranos-6-yl)ethane-1,2-diamine (6): A $25 \mathrm{~mL}$ round-bottom flask was charged with $0.30 \mathrm{~g}$ (0.54 mmol, 1.00 eq.) tert-butyl-\{2-[(1,7-dicarba-closo-dodecaboran-1-yl)methyl(1,2,:3,4-di-O-isopropylidene-6-deoxy- $\alpha$-D-galactopyranos-6-yl)amino]ethyl\}-carbamate (4) and $2.00 \mathrm{~mL}(26.0 \mathrm{mmol}, 48.2 \mathrm{eq}$.) TFA were added. The reaction mixture was stirred for $3 \mathrm{~h}$ at room temperature. The reaction was stopped by adding $4 \mathrm{~mL}$ DCM with subsequent evaporation of all volatile components under reduced pressure. This procedure was repeated three times. The resulting crude product was further purified by adding $4 \mathrm{~mL}$ saturated $\mathrm{NaHCO}_{3}$ solution and sonication for about $15 \mathrm{~min}$. Again, $3 \mathrm{~mL}$ DCM were added with stirring, under observation of gas evolution, and after 5 min the resulting layers were separated. The aqueous layer was extracted with $3 \mathrm{~mL}$ ethyl acetate. The combined organic layers were washed twice with $2 \mathrm{~mL}$ distilled water each. The organic layer was dried over $\mathrm{MgSO}_{4}$, the drying agents were filtered off and the solvent was removed under reduced pressure. Compound $6\left(0.25 \mathrm{~g}, 0.54 \mathrm{mmol}\right.$, quant., $R_{\mathrm{f}}=0.03$, nhexane/ethyl acetate, 5:1, (v/v)) was isolated as a colorless foamy solid. ${ }^{1} \mathrm{H}-\mathrm{NMR}(400 \mathrm{MHz}$, chloroform- $\left.d_{1}\right): \delta[\mathrm{ppm}]=1.33,1.34,1.45,1.54\left(\mathrm{~s}, 12 \mathrm{H}, 12,12^{\prime}, 13\right.$ and $\left.13^{\prime} \mathrm{CH}_{3}\right), 1.40$ to $3.16(\mathrm{~m}$, br, $10 \mathrm{H}, 10 \mathrm{BH}), 2.62$ to $2.69\left(\mathrm{~m}, 1 \mathrm{H},{ }^{7}\right.$ or $\left.{ }^{8} \mathrm{CHH}\right), 2.72$ to $2.77\left(\mathrm{~m}, 2 \mathrm{H},{ }^{7}\right.$ or $/$ and $\left.{ }^{8} \mathrm{CHH}\right), 2.77$ to $2.86\left(\mathrm{~m}, 3 \mathrm{H},{ }^{7}\right.$ or ${ }^{8} \mathrm{CHH}$ and $\left.{ }^{6} \mathrm{CH}_{2}\right), 2.92\left(\mathrm{~s}, 1 \mathrm{H},{ }^{16} \mathrm{CH}\right), 3.00\left(\mathrm{~d}, 1 \mathrm{H},{ }^{14} \mathrm{CHH},{ }^{2} \mathrm{~J}_{\mathrm{HH}}=15.4 \mathrm{~Hz}\right)$, $3.11\left(\mathrm{~d}, 1 \mathrm{H},{ }^{14} \mathrm{CHH},{ }^{2} J_{\mathrm{HH}}=15.4 \mathrm{~Hz}\right) 3.87\left(\mathrm{~m}, 1 \mathrm{H},{ }^{5} \mathrm{CH}\right), 4.17\left(\mathrm{dd}, 1 \mathrm{H},{ }^{4} \mathrm{CH},{ }^{3} J_{\mathrm{HH}}=7.9 \mathrm{~Hz}\right.$, $\left.{ }^{3} J_{\mathrm{HH}}=1.9 \mathrm{~Hz}\right), 4.30\left(\mathrm{dd}, 1 \mathrm{H},{ }^{2} \mathrm{CH},{ }^{3} \mathrm{~J}_{\mathrm{HH}}=5,2 \mathrm{~Hz},{ }^{3} J_{\mathrm{HH}}=2.4 \mathrm{~Hz}\right), 4.60\left(\mathrm{dd}, 1 \mathrm{H},{ }^{3} \mathrm{CH}\right.$, $\left.{ }^{3} J_{\mathrm{HH}}=7.9 \mathrm{~Hz},{ }^{3} J_{\mathrm{HH}}=2.4 \mathrm{~Hz}\right), 5.52\left(\mathrm{~d}, 1 \mathrm{H},{ }^{1} \mathrm{CH},{ }^{3} \mathrm{~J}_{\mathrm{HH}}=5.1 \mathrm{~Hz}\right) .{ }^{13} \mathrm{C}\left\{{ }^{1} \mathrm{H}\right\}-\mathrm{NMR}(100 \mathrm{MHz}$,

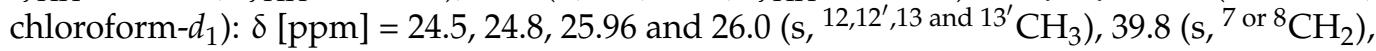


$53.2\left(\mathrm{~s},{ }^{6} \mathrm{CH}_{2}\right), 55.0\left(\mathrm{~s},{ }^{16} \mathrm{CH}\right), 57.7\left(\mathrm{~s},{ }^{7}\right.$ or $\left.{ }^{8} \mathrm{CH}_{2}\right), 60.2\left(\mathrm{~s},{ }^{14} \mathrm{CH}_{2}\right), 66.1\left(\mathrm{~s},{ }^{5} \mathrm{CH}\right), 70.3\left(\mathrm{~s},{ }^{2} \mathrm{CH}\right)$, $70.8\left(\mathrm{~s},{ }^{3} \mathrm{CH}\right), 72.0\left(\mathrm{~s},{ }^{4} \mathrm{CH}\right), 96.5\left(\mathrm{~s},{ }^{1} \mathrm{CH}\right), 108.5$ and $109.3\left(\mathrm{~s}, 10\right.$ and $\left.{ }^{11} \mathrm{C}_{\mathrm{q}}\right),{ }^{15} \mathrm{C}_{\mathrm{q}}$ was not observable (assumed at $77.7 \mathrm{ppm}) .{ }^{11} \mathrm{~B}\left\{{ }^{1} \mathrm{H}\right\}-\mathrm{NMR}\left(128 \mathrm{MHz}\right.$, chloroform- $\left.d_{1}\right): \delta[\mathrm{ppm}]=-15.6$ $(\mathrm{s}, 2 \mathrm{~B}),-13.5(\mathrm{~s}, 2 \mathrm{~B}),-11.2(\mathrm{~s}, 2 \mathrm{~B}),-10.8(\mathrm{~s}, 2 \mathrm{~B}),-9.3(\mathrm{~s}, 1 \mathrm{~B}),-4.3(\mathrm{~s}, 1 \mathrm{~B}) . \mathrm{IR}(\mathrm{KBr}): \tilde{\mathrm{v}}=2986$ $\left(\mathrm{w}, v \mathrm{CH}-\mathrm{sp}^{3}\right), 2933\left(\mathrm{w}, v \mathrm{CH}-\mathrm{sp}^{3}\right), 2593\left(\mathrm{~m}, v \mathrm{BH}-\mathrm{sp}^{3}\right), 1685\left(\mathrm{w}, \delta \mathrm{NH}-\mathrm{sp}^{3}\right), 1455\left(\mathrm{w}, \delta \mathrm{CH}-\mathrm{sp}^{3}\right)$, $1381\left(\mathrm{~m}, \delta \mathrm{CH}-\mathrm{sp}^{3}\right) \mathrm{cm}^{-1}$. ESI-HRMS: calculated for $\left[\mathrm{C}_{17} \mathrm{H}_{39} \mathrm{~B}_{10} \mathrm{~N}_{2} \mathrm{O}_{5}\right]^{+}=459.3862$; observed $459.3851[\mathrm{M}+\mathrm{H}]^{+}$; calculated for $\left[\mathrm{C}_{34} \mathrm{H}_{77} \mathrm{~B}_{20} \mathrm{~N}_{4} \mathrm{O}_{10}\right]^{+}=918.7724$; observed 918.7607 $[2 \mathrm{M}+\mathrm{H}]^{+}$.

$N^{1}$-[(1,7-dicarba-closo-dodecaboran-1-yl)methyl]- $N^{1}-(1,2: 3,4-d i$-O-isopropylidene-6-deoxy- $\alpha$ D-galacatopyranos-6-yl)-N ${ }^{2}$-[(4-methyl)-2-oxo-2H-chromen-7-yl)glycineamide (7): Method A. Under nitrogen atmosphere, $1 \mathrm{~mL}(0.98 \mathrm{~g}, 12.4 \mathrm{mmol})$ of pyridine was added to a mixture of N-[(1,7-dicarba-closo-dodecaboran-1-yl)methyl]-N-(1,2:3,4-di-O-isopropylidene-6deoxy- $\alpha$-D-galacatopyranos-6-yl)glycine (5) (100 mg, $0.21 \mathrm{mmol}, 1.00$ eq.) and 7-amino-4methylcoumarin $(44.4 \mathrm{mg}, 0.25 \mathrm{mmol}, 1.20$ eq.). After degassing the yellowish solution with nitrogen, phosphorus(V) oxychloride $(20.0 \mu \mathrm{L}, 0.23 \mathrm{mmol}, 1.10 \mathrm{eq}$.) was added dropwise at $-18{ }^{\circ} \mathrm{C}$. The solution immediately changed to a red suspension and after a while back to a yellow solution. The mixture was stirred for $1 \mathrm{~h}$ at $-18{ }^{\circ} \mathrm{C}$. After the reaction was finished, the mixture was poured into $\mathrm{H}_{2} \mathrm{O}$ and extracted with ethyl acetate. Subsequently, the combined organic layers were washed with a $\mathrm{NaHCO}_{3}$ solution and a saturated $\mathrm{NaCl}$ solution and then dried over $\mathrm{MgSO}_{4}$. After filtration, the solvent was removed under reduced pressure and the crude product was purified by column chromatography using n-hexane/ethyl acetate $\left(1: 1,(v / v), R_{\mathrm{f}}=0.51\right)$ as eluent. Compound 7 was obtained as a brownish solid in $27 \%$ yield ( $36 \mathrm{mg}, 5.71 \mu \mathrm{mol}$ ).

Method B. N-[(1,7-dicarba-closo-dodecaboran-1-yl)methyl]-N-(1,2:3,4-di-O-isopropylid ene-6-deoxy- $\alpha$-D-galacatopyranos-6-yl)glycine $(86.1 \mathrm{mg}, 0.18 \mathrm{mmol}, 1.00$ eq.) was added to a Schlenk tube and was dissolved in $5 \mathrm{~mL}$ absolute DCM. Subsequently, the solution was cooled to $0{ }^{\circ} \mathrm{C}$ in an ice bath and 7-amino-4-methylcoumarin $(39.1 \mathrm{mg}, 0.22 \mathrm{mmol}$, 1.23 eq.), HOBt (29.5 mg, $0.22 \mathrm{mmol}, 1.20$ eq.), EDCI (45.1 mg, $0.24 \mathrm{mmol}, 1.30$ eq.) and DIPEA ( $80 \mu \mathrm{L}, 0.46 \mathrm{mmol}, 2.54$ eq.) were added. Afterwards, the ice bath was removed, the reaction mixture was allowed to reach room temperature and stirred at room temperature for $22 \mathrm{~h}$. Subsequently, the solvent was removed under reduced pressure and the residue was redissolved in $20 \mathrm{~mL}$ ethyl acetate and washed with $30 \mathrm{~mL}$ of $1 \mathrm{~N} \mathrm{HCl}$, concentrated $\mathrm{NaHCO}_{3}$ and $\mathrm{NaCl}$ solution, respectively. The organic layer was dried over $\mathrm{Na}_{2} \mathrm{SO}_{4}$, filtered and the solvent was removed under reduced pressure. The obtained crude product was purified by column chromatography using $n$-hexane/ethyl acetate $\left(1: 1\left(R_{\mathrm{f}}=0.51\right)\right.$ to $1: 2,(v / v))$ as eluent, affording compound 7 as a sticky, brownish solid in $18 \%$ yield (20 $\mathrm{mg}$, $32 \mu \mathrm{mol}) .{ }^{1} \mathrm{H}-\mathrm{NMR}\left(400 \mathrm{MHz}\right.$, chloroform- $\left.d_{1}\right): \delta[\mathrm{ppm}]=1.28,1.29,1.38,1.59(\mathrm{~s}, 12 \mathrm{H}$, $12,12^{\prime}, 13$ and $\left.13^{\prime} \mathrm{CH}_{3}\right), 1.50$ and $3.00(\mathrm{~m}, \mathrm{br}, 10 \mathrm{H}, 10 \mathrm{BH}), 2.42\left(\mathrm{~d}, 3 \mathrm{H},{ }^{23} \mathrm{CH}_{3},{ }^{4} J_{\mathrm{HH}}=1.2 \mathrm{~Hz}\right)$, 2.86 to $2.97\left(\mathrm{~m}, 3 \mathrm{H},{ }^{6} \mathrm{CHH},{ }^{6} \mathrm{CHH}\right.$ and $\left.{ }^{16} \mathrm{CH}\right), 3.16\left(\mathrm{~d}, 1 \mathrm{H},{ }^{14} \mathrm{CHH},{ }^{2} \mathrm{~J}_{\mathrm{HH}}=15.5 \mathrm{~Hz}\right), 3.25$ $\left(\mathrm{d}, 1 \mathrm{H},{ }^{14} \mathrm{CHH},{ }^{2} J_{\mathrm{HH}}=15.5 \mathrm{~Hz}\right), 3.43\left(\mathrm{~d}, 1 \mathrm{H},{ }^{7} \mathrm{CHH},{ }^{2} J_{\mathrm{HH}}=17.5 \mathrm{~Hz}\right), 3.52\left(\mathrm{~d}, 1 \mathrm{H},{ }^{7} \mathrm{CH}\right.$, $\left.{ }^{2} J_{\mathrm{HH}}=17.4 \mathrm{~Hz}\right), 3.93\left(\mathrm{~m}, 1 \mathrm{H},{ }^{5} \mathrm{CH}, \mathrm{J}_{\mathrm{HH}}=9.7\right.$ and $\left.2.5 \mathrm{~Hz}\right), 4.10\left(\mathrm{dd}, 1 \mathrm{H},{ }^{4} \mathrm{CH},{ }^{3} \mathrm{~J}_{\mathrm{HH}}=7.8 \mathrm{~Hz}\right.$, $\left.{ }^{3} J_{\mathrm{HH}}=2.0 \mathrm{~Hz}\right), 4.41\left(\mathrm{dd}, 1 \mathrm{H},{ }^{2} \mathrm{CH},{ }^{3} \mathrm{~J}_{\mathrm{HH}}=5.2 \mathrm{~Hz},{ }^{3} \mathrm{~J}_{\mathrm{HH}}=2.4 \mathrm{~Hz}\right), 4.63\left(\mathrm{dd}, 1 \mathrm{H},{ }^{3} \mathrm{CH},{ }^{3} J_{\mathrm{HH}}=\right.$ $\left.7.8 \mathrm{~Hz},{ }^{3} J_{\mathrm{HH}}=2.4 \mathrm{~Hz}\right), 5.69\left(\mathrm{~d}, 1 \mathrm{H},{ }^{1} \mathrm{CH},{ }^{3} J_{\mathrm{HH}}=5.2 \mathrm{~Hz}\right), 6.21\left(\mathrm{~d}, 1 \mathrm{H},{ }^{21} \mathrm{CH},{ }^{4} J_{\mathrm{HH}}=1.4 \mathrm{~Hz}\right)$, 7.53 to $7.58\left(\mathrm{~m}, 2 \mathrm{H},{ }^{18} \mathrm{CH}\right.$ and $\left.{ }^{25} \mathrm{CH}\right), 7.71\left(\mathrm{dd}, 1 \mathrm{H},{ }^{26} \mathrm{CH},{ }^{3} \mathrm{~J}_{\mathrm{HH}}=8.7 \mathrm{~Hz},{ }^{4} \mathrm{~J}_{\mathrm{HH}}=2.0 \mathrm{~Hz}\right)$, $9.57\left(\mathrm{~s}, 1 \mathrm{H},{ }^{9} \mathrm{NH}\right) .{ }^{13} \mathrm{C}\left\{{ }^{1} \mathrm{H}\right\}-\mathrm{NMR}\left(100 \mathrm{MHz}\right.$, chloroform- $\left.d_{1}\right): \delta[\mathrm{ppm}]=18.6\left(\mathrm{~s},{ }^{23} \mathrm{CH}_{3}\right)$,

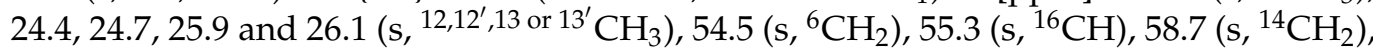
$60.0\left(\mathrm{~s},{ }^{7} \mathrm{CH}_{2}\right), 64.8\left(\mathrm{~s},{ }^{5} \mathrm{CH}\right), 70.3\left(\mathrm{~s},{ }^{2} \mathrm{CH}\right), 70.8\left(\mathrm{~s},{ }^{3} \mathrm{CH}\right), 71.7\left(\mathrm{~s},{ }^{4} \mathrm{CH}\right), 75.9\left(\mathrm{~s},{ }^{15} \mathrm{C}_{\mathrm{q}}\right), 96.5$ $\left(\mathrm{s},{ }^{1} \mathrm{CH}\right), 107.0\left(\mathrm{~s},{ }^{18} \mathrm{CH}\right), 108.9$ and $109.6\left(\mathrm{~s},{ }^{10}\right.$ and $\left.{ }^{11} \mathrm{C}_{\mathrm{q}}\right), 113.4\left(\mathrm{~s},{ }^{21} \mathrm{CH}\right), 115.7\left(\mathrm{~s},{ }^{26} \mathrm{CH}\right)$, $116.2\left(\mathrm{~s},{ }^{22} \mathrm{C}_{\mathrm{q}}\right), 125.2\left(\mathrm{~s},{ }^{25} \mathrm{CH}\right), 141.2\left(\mathrm{~s},{ }^{17} \mathrm{C}_{\mathrm{q}}\right), 152.3\left(\mathrm{~s},{ }^{24} \mathrm{C}_{\mathrm{q}}\right), 154.3\left(\mathrm{~s},{ }^{19} \mathrm{C}_{\mathrm{q}}\right), 161.2\left(\mathrm{~s},{ }^{20} \mathrm{C}_{\mathrm{q}}\right)$, $169.3\left(\mathrm{~s},{ }^{8} \mathrm{C}_{\mathrm{q}}\right) .{ }^{11} \mathrm{~B}\left\{{ }^{1} \mathrm{H}\right\}-\mathrm{NMR}\left(128 \mathrm{MHz}\right.$, chloroform- $\left.\mathrm{d}_{1}\right): \delta[\mathrm{ppm}]=-15.6(\mathrm{~s}, 2 \mathrm{~B}),-13.4(\mathrm{~s}$, 2B), $-11.2(\mathrm{~s}, 2 \mathrm{~B}),-10.5(\mathrm{~s}, 2 \mathrm{~B}),-8.9(\mathrm{~s}, 1 \mathrm{~B}),-4.4(\mathrm{~s}, 1 \mathrm{~B})$. IR $(\mathrm{KBr}): \tilde{v}=3288(\mathrm{~m}, v(\mathrm{NH}$, secondary amide (trans)), $3058\left(\mathrm{w}, v \mathrm{CH}-\mathrm{sp}^{2}\right), 2985\left(\mathrm{w}, \mathrm{v}_{\mathrm{as}} \mathrm{CH}_{2}-\mathrm{sp}^{3}\right), 2957\left(\mathrm{w}, \mathrm{v}_{\mathrm{s}} \mathrm{CH}_{2}-\mathrm{sp}^{3}\right)$, $2922\left(\mathrm{~m}, v_{\mathrm{as}} \mathrm{CH}_{3}-\mathrm{sp}^{3}\right), 2852\left(\mathrm{~m}, v_{\mathrm{s}} \mathrm{CH}_{3}-\mathrm{sp}^{3}\right), 2595\left(\mathrm{~m}, v B H-\mathrm{sp}^{3}\right), 1727$ (s, vC=O lactone $), 1706$ (s, vC $=\mathrm{O}_{\text {amide, }}$ amide I), $1616(v \mathrm{C}=\mathrm{C}, \alpha, \mathrm{B}$-unsaturation), $1567(\vee \mathrm{C}=\mathrm{C}$, aromatic or $\delta \mathrm{NH}$, 
amide II), 1519 (vC=C, aromatic or $v \mathrm{CN}$, amide II), $1454\left(\mathrm{~m}, \delta_{\mathrm{as}} \mathrm{CH}_{3}-\mathrm{sp}^{3}\right), 1369\left(\mathrm{~m}, \delta_{\mathrm{s}} \mathrm{CH}_{3}\right.$ $\left.\mathrm{sp}^{3}\right), 1066$ (s, vC-O-C $\left.\mathrm{Ether}\right), 901\left(\mathrm{~m}, \delta \mathrm{CH}-\mathrm{sp}^{2} /\right.$ aromatic), 853, $806\left(\mathrm{~m}, \delta \mathrm{CH}-\mathrm{sp}^{2} /\right.$ aromatic $)$ $\mathrm{cm}^{-1}$. ESI-HRMS: $(\mathrm{m} / z)$ calculated for $\left[\mathrm{NaC}_{27} \mathrm{H}_{42} \mathrm{~B}_{10} \mathrm{~N}_{2} \mathrm{O}_{8}\right]^{+}=653.3843$; observed 653.3840 $[\mathrm{M}+\mathrm{Na}]^{+}$.

$N^{2}$-(4-\{[(2-Amino-4-hydroxypteridine-6-yl)methyl]amino\}benzoyl)- $N^{5}-\{2-[(1,7$-dicarbacloso-dodecaboran-1-ylmethyl)-(1,2:3,4-di-O-isopropyliden-6-deoxy- $\alpha$-D-galactopyranos-6yl)amino]ethyl $\}$-L-glutamine (8) and (S)-2-(4-\{[(2-amino-4-hydroxypteridine-6-yl)methyl]am ino\}benzamido)- $N^{1}, N^{5}$-bis-\{2-[(1,7-dicarba-closo-dodecaborane-1-yl)methyl-(1,2:3,4-di-O-iso propylidene-6-deoxy- $\alpha$-D-galactopyranos-6-yl)amino]ethyl amino)pentanediamide (9): A $100 \mathrm{~mL}$ Schlenk flask was charged with $0.24 \mathrm{~g}$ ( $0.55 \mathrm{mmol}, 1.00$ eq.) folic acid and $20 \mathrm{~mL}$ dimethylformamide were added. The mixture was sonicated for $15 \mathrm{~min}$ and, subsequently, warmed to $37{ }^{\circ} \mathrm{C}$ for $15 \mathrm{~min}$ until a clear solution was obtained. To this mixture, $0.11 \mathrm{~g}$ ( $0.55 \mathrm{mmol}, 1.00$ eq.) $\mathrm{DCC}$ and $0.06 \mathrm{~g}$ (0.55 mmol, 1.00 eq.) NHS were added. The reaction mixture was stirred for $16 \mathrm{~h}$ at room temperature. Afterwards, $0.25 \mathrm{~g}$ (0.55 mmol, 1.00 eq.) $N^{1}$-[(1,7-dicarba-closo-dodecaborane-1-yl)methyl]- $N^{1}$-(1,2:3,4-di-Oisopropylidene-6-deoxy- $\alpha$-D-galactopyranos-6-yl)ethane-1,2-diamine (6), dissolved in $7 \mathrm{~mL}$ dimethylformamide, were added to this solution and the mixture was stirred overnight at room temperature. The resulting suspension was filtered under inert conditions. Subsequently, $0.10 \mathrm{~mL}$ ( $0.61 \mathrm{mmol}, 1.10$ eq.) $\mathrm{N}, \mathrm{N}$-diisopropylethylamine were added and the mixture was stirred overnight at room temperature. The reaction was stopped by adding $50 \mathrm{~mL}$ ice-cold diethyl ether. Simultaneously, the mixture was cooled in an ice bath. Completion of the precipitation was achieved by storage for one additional night at $-20{ }^{\circ} \mathrm{C}$. The resulting precipitate was filtered off and washed with $10 \mathrm{~mL}$ ice-cold diethyl ether. The precipitate was suspended in $5 \mathrm{~mL}$ diethyl ether and subsequently sonicated for $15 \mathrm{~min}$. Afterwards, the raw product was filtered again. The orange-red solid was washed with $5 \mathrm{~mL}$ diethyl ether and the previously described procedure was repeated one more time. Afterwards, the precipitate was dried in vacuo. It was not possible to isolate the desired compound $\mathbf{8}$, but mass spectrometry revealed the presence of $N^{2}$-(4-\{[(2-amino-4-hydroxypteridine-6-yl)methyl]amino\}benzoyl)- $N^{5}-\{2-[(1,7$-dicarbacloso-dodecaboran-1-yl)methyl-(1,2:3,4-di-O-isopropyliden-6-deoxy- $\alpha$-D-galactopyranos-6yl)amino]ethyl\}-L-glutamine (8) and (S)-2-(4-\{[(2-amino-4-hydroxypteridine-6-yl)methyl]am ino\}benzamido)- $N^{1}, N^{5}$-bis-\{2-[(1,7-dicarba-closo-dodecaborane-1-yl)methyl-(1,2:3,4-di-O-iso propylidene-6-deoxy- $\alpha$-D-galactopyranos-6-yl)amino]ethyl\}amino)pentanediamide (9). Due to impurities, it was not possible to determine a yield based on HRMS. 8: ESI-HRMS: $(\mathrm{m} / \mathrm{z})$ calculated for $\left[\mathrm{C}_{36} \mathrm{H}_{56} \mathrm{~B}_{10} \mathrm{~N}_{9} \mathrm{O}_{10}\right]^{+}=883.5139$; observed $883.5151[\mathrm{M}+\mathrm{H}]^{+}$; calculated for $\left[\mathrm{NaC}_{36} \mathrm{H}_{55} \mathrm{~B}_{10} \mathrm{~N}_{9} \mathrm{O}_{10}\right]^{+}=905.4959$; observed $905.4963[\mathrm{M}+\mathrm{Na}]^{+}$; calculated for $\left[\mathrm{KC}_{36} \mathrm{H}_{55} \mathrm{~B}_{10} \mathrm{~N}_{9} \mathrm{O}_{10}\right]^{+}=921.4700 ;$ observed $921.4715[\mathrm{M}+\mathrm{K}]^{+}$. 9:1E7D IR $(\mathrm{KBr}): \tilde{v}=3315$ $\left(\mathrm{w}, v \mathrm{NH}-\mathrm{sp}^{3}\right), 2931\left(\mathrm{w}, v \mathrm{CH}-\mathrm{sp}^{3}\right), 2850\left(\mathrm{w}, v \mathrm{CH}-\mathrm{sp}^{3}\right), 2594\left(\mathrm{w}, v \mathrm{BH}-\mathrm{sp}^{3}\right), 1723(\mathrm{~m}$, amide I), $1687\left(\mathrm{~m}\right.$, amide II), $1605\left(\mathrm{~s}, v \mathrm{C}=\mathrm{C}_{\text {arom. }}\right), 1412\left(\mathrm{~m}, \delta \mathrm{CH}-\mathrm{sp}^{3}\right) \mathrm{cm}^{-1}$. ESI-HRMS: $(m / z)$ calculated for $\left[\mathrm{C}_{53} \mathrm{H}_{92} \mathrm{~B}_{20} \mathrm{~N}_{11} \mathrm{O}_{14}\right]^{+}=1323.8830$; observed $1323.8829[\mathrm{M}+\mathrm{H}]^{+}$; calculated for $\left[\mathrm{NaC}_{53} \mathrm{H}_{91} \mathrm{~B}_{20} \mathrm{~N}_{11} \mathrm{O}_{14}\right]^{+}=1345.8649$; observed $1345.8625[\mathrm{M}+\mathrm{Na}]^{+}$; calculated for $\left[\mathrm{KC}_{53} \mathrm{H}_{91} \mathrm{~B}_{20} \mathrm{~N}_{11} \mathrm{O}_{14}\right]^{+}=1361.8390$; observed $1361.8349[\mathrm{M}+\mathrm{K}]^{+}$.

\section{Conclusions}

In this work we reported the successful design of a novel modular, small-moleculebased approach to synthesizing boron-rich compounds bearing a carboxylic acid group or a primary amine group as potential coupling partners for suitable tumor-selective biomolecules. As proof of concept, conjugates with 7-amino-4-methylcoumarin and folic acid were obtained. While the present work focused on the development of a synthetic protocol, the next steps will include the deprotection of the respective galactopyranosyl protecting groups under acidic aqueous conditions [61] followed by biological investigations.

Supplementary Materials: Supplementary information is available online, including the numbering scheme of the isolated compounds 1-7, NMR spectra of compounds 5, 6 and 7 and mass spectra 
of 8 and 9, additional synthetic procedures and analytical data for $\mathbf{2}^{\prime}$, ESI-3, ESI-3', ESI-4 and 1(trifluoromethanesulfonylmethyl)-1,7-dicarba-closo-dodecaborane(12), crystallographic information for compound ESI-3' ${ }^{\prime}$, information about the exploration of the optimization for the synthesis of 3 and the deprotection protocol for 4 , and the extension of the synthetic protocol to ortho-carborane derivatives.

Author Contributions: Conceptualization, M.K. and E.H.-H.; Data curation, M.K. and P.L.; formal analysis, M.K., J.-S.J.F., A.F., N.A.U., J.T. and P.L.; funding acquisition, E.H.-H.; investigation, M.K., J.-S.J.F., A.F., N.A.U. and J.T.; methodology, M.K.; project administration, M.K. and E.H.-H.; resources, E.H.-H.; supervision, E.H.-H.; validation, M.K., J.-S.J.F., A.F., N.A.U. and J.T.; visualization, M.K.; writing—original draft, M.K.; writing—review \& editing, J.S.J.F., A.F., N.A.U., J.T., P.L. and E.H.-H. All authors have read and agreed to the published version of the manuscript.

Funding: This research was funded by the Europäischer Fonds für regionale Entwicklung (EFRE), the Free State of Saxony (ESF) and the Leipzig School of Natural Sciences-Building with Molecules and Nano-objects (BuildMoNa).

Institutional Review Board Statement: Not applicable.

Informed Consent Statement: Not applicable.

Data Availability Statement: The data presented in this study are available on request from the corresponding author.

Acknowledgments: We thank Ramona Oehme, Susann Billig and Claudia Birkemeyer for measuring the mass spectra and Ines Rein, Stefanie Märcker-Recklies and Jaqueline Lewandowski for recording the infrared spectra.

Conflicts of Interest: The authors declare no conflict of interest.

Sample Availability: Samples of the compounds are not available from the authors.

\section{References}

1. Locher, G.L. Biological Effects and Therapeutic Possibilities of Neutrons. Am. J. Roentgenol. Radi. 1936, 36, 1-18.

2. Hatanaka, H. A Revised Boron-Neutron Capture Therapy for Malignant Brain Tumors. J. Neurol. 1975, 209, 81-94. [CrossRef] [PubMed]

3. Taylor, H.J.; Goldhaber, M. Detection of Nuclear Disintegration in a Photographic Emulsion. Nature 1935, 135, 341. [CrossRef]

4. Chadwick, J.; Goldhaber, M. Disintegration by Slow Neutrons. Nature 1935, 135, 65. [CrossRef]

5. Barth, R.F.; Soloway, A.H.; Fairchild, R.G. Boron Neutron Capture Therapy of Cancer. Cancer Res. 1990, 50, 1061-1070. [CrossRef]

6. Soloway, A.H.; Tjarks, W.; Barnum, B.A.; Rong, F.-G.; Barth, R.F.; Codogni, I.M.; Wilson, J.G. The Chemistry of Neutron Capture Therapy. Chem. Rev. 1998, 98, 1515-1562. [CrossRef]

7. Hartman, T.; Carlsson, J. Radiation Dose Heterogeneity in Receptor and Antigen Mediated Boron Neutron Capture Therapy. Radiother. Oncol. 1994, 31, 61-75. [CrossRef]

8. Coderre, J.A.; Turcotte, J.C.; Riley, K.J.; Binns, P.J.; Harling, O.K.; Kiger, W.S. Boron Neutron Capture Therapy: Cellular Targeting of High Linear Energy Transfer Radiation. Technol. Cancer Res. Treat. 2003, 2, 355-375. [CrossRef]

9. Sears, V.F. Neutron Scattering Lengths and Cross Sections. Neutron News 1992, 3, 26-37. [CrossRef]

10. Hawthorne, M.F. The Role of Chemistry in the Development of Boron Neutron Capture Therapy of Cancer. Angew. Chem. Int. Ed. 1993, 32, 950-984. [CrossRef]

11. Adams, L.; Tomlinson, S.; Wang, J.; Hosmane, S.N.; Maguire, J.A.; Hosmane, N.S. Novel Approach to Boron-10 Enriched Decaborane(14): An Important Advance in Synthetic Boron Hydride Chemistry. Inorg. Chem. Commun. $2002,5,765-767$. [CrossRef]

12. Yinghuai, Z.; Widjaja, E.; Lo Sia, S.P.; Zhan, W.; Carpenter, K.; Maguire, J.A.; Hosmane, N.S.; Hawthorne, M.F. Ruthenium(0) Nanoparticle-Catalyzed Isotope Exchange between ${ }^{10} \mathrm{~B}$ and ${ }^{11} \mathrm{~B}$ Nuclei in Decaborane(14). J. Am. Chem. Soc. 2007, 129, 6507-6512. [CrossRef] [PubMed]

13. Chadwick, J. Possible Existence of a Neutron. Nature 1932, 129, 312. [CrossRef]

14. Petry, W.; Neuhaus, J. Neutronen nach Maß. Phys. J. 2007, 6, 31-37.

15. Kettenbach, K.; Schieferstein, H.; Grunewald, C.; Iffland, D.; Reffert, L.M.; Hampel, G.; Schütz, C.L.; Bings, N.H.; Ross, T.L. Synthesis and Evaluation of Boron Folates for Boron-Neutron-Capture-Therapy (BNCT). Radiochim. Acta 2015, 103, 799-809. [CrossRef]

16. Rong, F.-G.; Soloway, A.H.; Ikeda, S.; Ives, D.H. Synthesis and Biochemical Activity of Hydrophilic Carborane-Containing Pyrimidine Nucleosides as Potential Agents for DNA Incorporation and BNCT. Nucleos. Nucleot. 1997, 16, 379-401. [CrossRef] 
17. Kueffer, P.J.; Maitz, C.A.; Khan, A.A.; Schuster, S.A.; Shlyakhtina, N.I.; Jalisatgi, S.S.; Brockman, J.D.; Nigg, D.W.; Hawthorne, M.F. Boron Neutron Capture Therapy Demonstrated in Mice Bearing EMT6 Tumors Following Selective Delivery of Boron by Rationally Designed Liposomes. Proc. Natl. Acad. Sci. USA 2013, 110, 6512-6517. [CrossRef]

18. Ahrens, V.M.; Frank, R.; Boehnke, S.; Schütz, C.L.; Hampel, G.; Iffland, D.S.; Bings, N.H.; Hey-Hawkins, E.; Beck-Sickinger, A.G. Receptor-mediated Uptake of Boron-rich Neuropeptide Y Analogues for Boron Neutron Capture Therapy. ChemMedChem 2015, 10, 164-172. [CrossRef]

19. Calabrese, G.; Daou, A.; Barbu, E.; Tsibouklis, J. Towards Carborane-functionalised Structures for the Treatment of Brain Cancer. Drug Discov. Today 2018, 23, 63-75. [CrossRef]

20. Barth, R.F.; Mi, P.; Yang, W. Boron Delivery Agents for Neutron Capture Therapy of Cancer. Cancer Commun. 2018, $38,35$. [CrossRef] [PubMed]

21. Bleuel, D.L.; Donahue, R.J.; Ludewigt, B.A.; Vujic, J. Designing Accelerator-based Epithermal Neutron Beams for Boron Neutron Capture Therapy. Med. Phys. 1998, 25, 1725-1734. [CrossRef] [PubMed]

22. Onishi, T.; Kumada, H.; Takada, K.; Naito, F.; Kurihara, T.; Sakae, T. Investigation of the Neutron Spectrum Measurement Method for Dose Evaluation in Boron Neutron Capture Therapy. Appl. Radiat. Isot. 2018, 140, 5-11. [CrossRef]

23. Durisi, E.; Alikaniotis, K.; Borla, O.; Bragato, F.; Costa, M.; Giannini, G.; Monti, V.; Visca, L.; Vivaldo, G.; Zanini, A. Design and Simulation of an Optimized E-linac Based Neutron Source for BNCT Research. Appl. Radiat. Isot. 2015, 106, 63-67. [CrossRef] [PubMed]

24. Kato, T.; Hirose, K.; Tanaka, H.; Mitsumoto, T.; Motoyanagi, T.; Arai, K.; Harada, T.; Takeuchi, A.; Kato, R.; Yajima, S.; et al. Design and Construction of an Accelerator-based Boron Neutron Capture Therapy (AB-BNCT) Facility with Multiple Treatment Rooms at the Southern Tohoku BNCT Research Center. Appl. Radiat. Isot. 2019, 156, 108961. [CrossRef]

25. Mori, Y.; Suzuki, A.; Yoshino, K.; Kakihana, H. Complex Formation of $p$-Boronophenylalanine with some Monosaccharides. Pigment Cell Res. 1989, 2, 273-277. [CrossRef]

26. Stella Pharma Corporation. Available online: https:/ / stella-pharma.co.jp/en (accessed on 6 March 2021).

27. Stella Pharma Corporation. News Release: STELLA PHARMA will Launch Steboronine ${ }^{\circledR}$, the World's First BNCT Drug, on May 20, 2020. Available online: https:/ /stella-pharma.co.jp/cp-bin/wordpress5/wp-content/uploads/2020/05/Steboroninelaunched_ENG.pdf (accessed on 6 March 2021).

28. Frank, R.; Ahrens, V.M.; Boehnke, S.; Beck-Sickinger, A.G.; Hey-Hawkins, E. Charge-Compensated Metallacarborane Building Blocks for Conjugation with Peptides. ChemBioChem 2016, 17, 308-317. [CrossRef] [PubMed]

29. Worm, D.J.; Hoppenz, P.; Els-Heindl, S.; Kellert, M.; Kuhnert, R.; Saretz, S.; Köbberling, J.; Riedl, B.; Hey-Hawkins, E.; BeckSickinger, A.G. Selective Neuropeptide Y Conjugates with Maximized Carborane Loading as Promising Boron Delivery Agents for Boron Neutron Capture Therapy. J. Med. Chem. 2020, 63, 2358-2371. [CrossRef]

30. Hoppenz, P.; Els-Heindl, S.; Kellert, M.; Kuhnert, R.; Saretz, S.; Lerchen, H.-G.; Köbberling, J.; Riedl, B.; Hey-Hawkins, E.; Beck-Sickinger, A.G. A Selective Carborane-Functionalized Gastrin-Releasing Peptide Receptor Agonist as Boron Delivery Agent for Boron Neutron Capture Therapy. J. Org. Chem. 2020, 85, 1446-1457. [CrossRef]

31. Hattori, Y.; Kusaka, S.; Mukumoto, M.; Uehara, K.; Asano, T.; Suzuki, M.; Masunaga, S.; Ono, K.; Tanimori, S.; Kirihata, M. Biological Evaluation of Dodecaborate-Containing L-Amino Acids for Boron Neutron Capture Therapy. J. Med. Chem. 2012, 55, 6980-6984. [CrossRef]

32. Pan, X.Q.; Wang, H.; Shukla, S.; Sekido, M.; Adams, D.M.; Tjarks, W.; Barth, R.F.; Lee, R.J. Boron-Containing Folate ReceptorTargeted Liposomes as Potential Delivery Agents for Neutron Capture Therapy. Bioconjug. Chem. 2002, 13, 435-442. [CrossRef]

33. Dubey, R.; Kushal, S.; Mollard, A.; Vojtovich, L.; Oh, P.; Levin, M.D.; Schnitzer, J.E.; Zharov, I.; Olenyuk, B.Z. Tumor Targeting, Trifunctional Dendritic Wedge. Bioconjug. Chem. 2015, 26, 78-89. [CrossRef] [PubMed]

34. Doi, A.; Kawabata, S.; Iida, K.; Yokoyama, K.; Kajimoto, Y.; Kuroiwa, T.; Shirakawa, T.; Kirihata, M.; Kasaoka, S.; Maruyama, K.; et al. Tumor-specific Targeting of Sodium Borocaptate (BSH) to Malignant Glioma by Transferrin-PEG Liposomes: A Modality for Boron Neutron Capture Therapy. J. Neurooncol. 2008, 87, 287-294. [CrossRef]

35. Mier, W.; Gabel, D.; Haberkorn, U.; Eisenhut, M. Conjugation of the closo-Borane Mercaptoundecahydrododecaborate (BSH) to a Tumour Selective Peptide. Z. Anorg. Allg. Chem. 2004, 630, 1258-1262. [CrossRef]

36. Liu, L.; Barth, R.F.; Adams, D.M.; Soloway, A.H.; Reisfeld, R.A. Bispecific Antibodies as Targeting Agents for Boron Neutron Capture Therapy of Brain Tumors. J. Hematother. 1995, 4, 477-483. [CrossRef] [PubMed]

37. Orlova, A.V.; Zinin, A.I.; Malysheva, N.N.; Kononov, L.O.; Sivaev, I.B.; Bregadze, V.I. Conjugates of Polyhedral Boron Compounds with Carbohydrates. 1. New Approach to the Design of Selective Agents for Boron Neutron Capture Therapy of Cancer. Russ. Chem. Bull. Int. Ed. 2003, 52, 2766-2769. [CrossRef]

38. Marepally, S.R.; Yao, M.-L.; Kabalka, G.W. Boronated Carbohydrate Derivatives as Potential Boron Neutron Capture Therapy Reagents. Future Med. Chem. 2013, 5, 693-704. [CrossRef] [PubMed]

39. Varadarajan, A.; Hawthorne, M.F. Novel Carboranyl Amino Acids and Peptides: Reagents for Antibody Modification and Subsequent Neutron-Capture Studies. Bioconjug. Chem. 1991, 2, 242-253. [CrossRef] [PubMed]

40. Timofeev, S.V.; Bregadze, V.I.; Osipov, S.N.; Titanyuk, I.D.; Petrovskii, P.V.; Starikova, Z.A.; Glukhov, I.V.; Beletskaya, I.P. New Carborane-containing Amino Acids and Their Derivatives. Crystal Structures of N-protected Carboranylalaninates. Russ. Chem. Bull. Int. Ed. 2007, 56, 791-797. [CrossRef] 
41. Stogniy, M.Y.; Zakharova, M.V.; Sivaev, I.B.; Godovikov, I.A.; Chizov, A.O.; Bregadze, V.I. Synthesis of New Carborane-based Amino Acids. Polyhedron 2013, 55, 117-120. [CrossRef]

42. Michiue, H.; Sakurai, Y.; Kondo, N.; Kitamatsu, M.; Bin, F.; Nakajima, K.; Hirota, Y.; Kawabata, S.; Nishiki, T.; Ohmori, I.; et al. The Acceleration of Boron Neutron Capture Therapy Using Multi-linked Mercaptoundecahydrododecaborate (BSH) Fused Cell-penetrating Peptide. Biomaterials 2014, 35, 3396-3405. [CrossRef] [PubMed]

43. Worm, D.J.; Els-Heindl, S.; Kellert, M.; Kuhnert, R.; Saretz, S.; Koebberling, J.; Riedl, B.; Hey-Hawkins, E.; Beck-Sickinger, A.G. A Stable meta-Carborane Enables the Generation of Boron-rich Peptide Agonists Targeting the Ghrelin Receptor. J. Pept. Sci. 2018, 32, e3119. [CrossRef]

44. Lerouge, F.; Viñas, C.; Teixidor, F.; Núñez, R.; Abreu, A.; Xochitiotzi, E.; Santillan, R.; Farfán, N. High Boron Content Carboranylfunctionalized Aryl Ether Derivatives Displaying Photoluminescent Properties. Dalton Trans. 2007, 92, 1898-1903. [CrossRef]

45. Feng, B.; Tomizawa, K.; Michiue, H.; Miyatake, S.-I.; Han, X.-J.; Fujimura, A.; Seno, M.; Kirihata, M.; Matsui, H. Delivery of Sodium Borocaptate to Glioma Cells Using Immunoliposome Conjugated with Anti-EGFR Antibodies by ZZ-His. Biomaterials 2009, 30, 1746-1755. [CrossRef]

46. Ciofani, G.; Raffa, V.; Menciassi, A.; Cuschieri, A. Folate Functionalized Boron Nitride Nanotubes and their Selective Uptake by Glioblastoma Multiforme Cells: Implications for their Use as Boron Carriers in Clinical Boron Neutron Capture Therapy. Nanoscale Res. Lett. 2008, 4, 113-121. [CrossRef]

47. Kellert, M.; Worm, D.J.; Hoppenz, P.; Sárosi, M.B.; Lönnecke, P.; Riedl, B.; Koebberling, J.; Beck-Sickinger, A.G.; Hey-Hawkins, E. Modular Triazine-based Carborane-containing Carboxylic Acids-Synthesis and Characterisation of Potential Boron Neutron Capture Therapy Agents Made of Readily Accessible Building Blocks. Dalton Trans. 2019, 48, 10834-10844. [CrossRef]

48. Kellert, M.; Hoppenz, P.; Lönnecke, P.; Worm, D.J.; Riedl, B.; Koebberling, J.; Beck-Sickinger, A.G.; Hey-Hawkins, E. Tuning a Modular System-Synthesis and Characterisation of a Boron-rich s-Triazine-based Carboxylic Acid and Amine Bearing a Galactopyranosyl Moiety. Dalton Trans. 2020, 49, 57-69. [CrossRef] [PubMed]

49. Kellert, M.; Lönnecke, P.; Riedl, B.; Koebberling, J.; Hey-Hawkins, E. Enlargement of a Modular System-Synthesis and Characterization of an s-Triazine-based Carboxylic Acid Ester Bearing a Galactopyranosyl Moiety and an Enormous Boron Load. Molecules 2019, 24, 3288. [CrossRef]

50. He, H.; Li, D.-W.; Yang, L.-Y.; Fu, L.; Zhu, X.-J.; Wong, W.-K.; Jiang, F.-L.; Liu, Y. A Novel Bifunctional Mitochondria-targeted Anticancer Agent with High Selectivity for Cancer Cells. Sci. Rep. 2015, 5, 13543. [CrossRef] [PubMed]

51. Bryden, F.; Savoie, H.; Rosca, E.V.; Boyle, R.W. PET/PDT Theranostics: Synthesis and Biological Evaluation of a Peptide-targeted Gallium Porphyrin. Dalton Trans. 2015, 44, 4925-4932. [CrossRef]

52. Griffith, D.; Morgan, M.P.; Marmion, C.J. A Novel Anti-cancer Bifunctional Platinum Drug Candidate with Dual DNA Binding and Histone Deacetylase Inhibitory Activity. Chem. Comm. 2009, 6735-6737. [CrossRef]

53. Chen, L.; Wang, X.; Ji, F.; Bao, Y.; Wang, J.; Guo, L.; Li, Y. New Bifunctional-pullulan-based Micelles with Good Biocompatibility for Efficient Co-delivery of Cancer-suppressing p53 Gene and Doxorubicin to Cancer Cells. RSC Adv. 2015, 5, 94719-94731. [CrossRef]

54. Soni, R.; Soman, S.S. Design and Synthesis of Aminocoumarin Derivatives as DPP-IV Inhibitors and Anticancer Agents. Bioorg. Chem. 2018, 79, 277-284. [CrossRef]

55. Achilli, C.; Jadhav, S.A.; Guidetti, G.F.; Ciana, A.; Abbonante, V.; Malara, A.; Fagnoni, M.; Torti, M.; Balduini, A.; Balduini, C.; et al. Folic Acid-Conjugated 4-Amino-Phenylboronate, a Boron-Containing Compound Designed for Boron Neutron Capture Therapy, is an Unexpected Agonist for Human Neutrophils and Platelets. Chem. Biol. Drug Des. 2014, 83, 532-540. [CrossRef] [PubMed]

56. Assaraf, Y.G.; Leamon, C.P.; Reddy, J.A. The Folate Receptor as a Rational Therapeutic Target for Personalized Cancer Treatment. Drug Resist. Updat. 2014, 17, 89-95. [CrossRef] [PubMed]

57. Goto, T.; Ohta, K.; Suzuki, T.; Ohta, S.; Endo, Y. Design and Synthesis of Novel Androgen Receptor Antagonists with Sterically Bulky Icosahedral Carboranes. Bioorgan. Med. Chem. 2005, 13, 6414-6424. [CrossRef] [PubMed]

58. Kalinin, V.N.; Rys, E.G.; Tyutyunov, A.A.; Starikova, Z.A.; Korlyukov, A.A.; Ol'shevskaya, V.A.; Sung, D.D.; Ponomaryov, A.B.; Petrovskii, P.V.; Hey-Hawkins, E. The First Carborane Triflates: Synthesis and Reactivity of 1-Trifluoromethanesulfonylmethyland 1,2-Bis(trifluoromethanesulfonylmethyl)-o-carborane. Dalton Trans. 2005, 903-908. [CrossRef]

59. Saltan, F.; Akat, H. Synthesis and Thermal Degradation Kinetics of D-(+)-Galactose Containing Polymers. Polimeros 2013, 23, 697-704. [CrossRef]

60. Brackhagen, M.; Boye, H.; Vogel, C. Synthesis of $\left(6^{2}-\mathrm{H}\right)$ - and 6-Deoxy-6-fluoro-L-galactose Derivatives. J. Carbohyd. Chem. 2001, 20, 31-43. [CrossRef]

61. Wuts, P.G.M.; Greene, T.W. Greene's Protective Groups in Organic Synthesis, 4th ed.; Wiley-Interscience: Hoboken, NJ, USA, 2007; ISBN 9780471697541.

62. Kokotos, G.; Theodorou, V.; Tzougraki, C. Synthesis and In Vitro Cytotoxicity of Aminocoumarin Platinum(II) Complexes. Bioorg. Med. Chem. Lett. 1997, 7, 2165-2168. [CrossRef]

63. Kaur, M.; Kohli, S.; Sandhu, S.; Bansal, Y.; Bansal, G. Coumarin: A Promising Scaffold for Anticancer Agents. Anticancer Agents Med. Chem. 2015, 15, 1032-1048. [CrossRef]

64. Klenkar, J.; Molnar, M. Natural and Synthetic Coumarins as Potential Anticancer Agents. J. Chem. Pharm. Res. 2015, 7, 1223-1238. 
65. Majnooni, M.B.; Fakhri, S.; Smeriglio, A.; Trombetta, D.; Croley, C.R.; Bhattacharyya, P.; Sobarzo-Sánchez, E.; Farzaei, M.H.; Bishayee, A. Antiangiogenic Effects of Coumarins against Cancer: From Chemistry to Medicine. Molecules 2019, $24,4278$. [CrossRef]

66. Shukla, S.; Wu, G.; Chatterjee, M.; Yang, W.; Sekido, M.; Diop, L.A.; Müller, R.; Sudimack, J.J.; Lee, R.J.; Barth, R.F.; et al. Synthesis and Biological Evaluation of Folate Receptor-targeted Boronated PAMAM Dendrimers as Potential Agents for Neutron Capture Therapy. Bioconjug. Chem. 2003, 14, 158-167. [CrossRef]

67. Cheung, A.; Bax, H.J.; Josephs, D.H.; Ilieva, K.M.; Pellizzari, G.; Opzoomer, J.; Bloomfield, J.; Fittall, M.; Grigoriadis, A.; Figini, M.; et al. Targeting Folate Receptor Alpha for Cancer Treatment. Oncotarget 2016, 7, 52553-52574. [CrossRef]

68. Quéléver, G.; Burlet, S.; Garino, C.; Pietrancosta, N.; Laras, Y.; Kraus, J.-L. Simple Coupling Reaction between Amino Acids and Weakly Nucleophilic Heteroaromatic Amines. J. Comb. Chem. 2004, 6, 695-698. [CrossRef]

69. El-Faham, A.; Albericio, F. Peptide Coupling Reagents, More than a Letter Soup. Chem. Rev. 2011, 111, 6557-6602. [CrossRef] [PubMed]

70. Trindade, A.F.; Frade, R.F.M.; Maçôas, E.M.S.; Graça, C.; Rodrigues, C.A.B.; Martinho, J.M.G.; Afonso, C.A.M. “Click and Go”: Simple and fast Folic Acid Conjugation. Org. Biomol. Chem. 2014, 12, 3181-3190. [CrossRef] [PubMed]

71. Nakamura, H.; Aoyagi, K.; Yamamoto, Y. Tetrabutylammonium Fluoride Promoted Novel Reactions of $o$-Carborane: Interand Intramolecular Additions to Aldehydes and Ketones and Annulation via Enals and Enones. J. Am. Chem. Soc. 1998, 120, 1167-1171. [CrossRef]

72. Tiwari, N.; Sharma, R.K.; Gupta, P.; Misra, S.; Misra-Bhattacharya, S.; Butcher, R.J.; Singh, K.; Katiyar, D. Synthesis, Structure Elucidation, Homology Modeling and Antifilarial Activity of 7-Benzamidocoumarin Derivatives. ChemistrySelect 2019, 4, 33003307. [CrossRef]

73. Harris, R.K.; Becker, E.D.; de Menezes, S.M.C.; Goodfellow, R.; Granger, P. NMR Nomenclature: Nuclear Spin Properties and Conventions for Chemical Shifts. IUPAC Recommendations 2001. Pure Appl. Chem. 2001, 73, 1795-1818. [CrossRef]

74. MestReNova, v12.00-20080; Mestrelab Research S.L.: Santiago de Compostela, Spain, 2017. 